\title{
Intuitionistic Fuzzy Semi HX Ring
}

\author{
R. Muthuraj ${ }^{1}$, M. S. Muthuraman ${ }^{2}$, K. H. Manikandan ${ }^{3}$ \\ ${ }^{I}$ Assistant Professor, P.G. Research Dept., of Mathematics, H. H. Rajahs' College, \\ Bharathidasan University, Pudukkottai, Tamil Nadu, India. \\ ${ }^{2}$ Associate Professor, Dept., of Mathematics, P.S.N.A.College of Engg., \& Tech., \\ Anna University, Dindigul, Tamil Nadu, India. \\ ${ }^{3}$ Assistant Professor, Dept., of Mathematics, P.S.N.A.College of Engg., \& Tech., \\ Anna University, Dindigul, Tamil Nadu, India.
}

*Corresponding Author: K. H. Manikandan, Assistant Professor, Dept., of Mathematics, P.S.N.A.College of Engg., \& Tech., Anna University, Dindigul, Tamil Nadu, India.

\begin{abstract}
In this paper, we define the notion of intuitionistic fuzzy semi HX ring of a semi HX ring and some of their related properties are investigated. We define the necessity and possibility operators of an intuitionistic fuzzy subset of an intuitionistic fuzzy semi HX ring and discuss some of its properties. We introduce the concept of an image, pre-image of an intuitionistic fuzzy subset and discuss in detail a series of homomorphic and anti homomorphic properties of an intuitionistic fuzzy set are discussed.
\end{abstract}

Keywords: Intuitionistic fuzzy set, fuzzy semi HX ring, intuitionistic fuzzy semi HX ring, homomorphism and anti homomorphism of an intuitionistic fuzzy semi HX ring, image and pre-image of an intuitionistic fuzzy set

\section{INTRODUCTION}

In 1965, Zadeh [12] introduced the concept of fuzzy subset $\mu$ of a set $X$ as a function from $X$ into the closed unit interval $[0,1]$ and studied their properties. Fuzzy set theory is a useful tool to describe situations in which the data or imprecise or vague and it is applied to logic, set theory, group theory, ring theory, real analysis, measure theory etc. In 1967, Rosenfeld [11] defined the idea of fuzzy subgroups and gave some of its properties. Li Hong Xing [5] introduced the concept of HX group. In 1982 Wang-jin Liu[7] introduced the concept of fuzzy ring and fuzzy ideal. With the successful upgrade of algebraic structure of group many researchers considered the algebraic structure of some other algebraic systems in which ring was considered as first. In 1988, Professor Li Hong Xing [6] proposed the concept of HX ring and derived some of its properties, then Professor Zhong [2,3] gave the structures of HX ring on a class of ring. R.Muthuraj et.al[10]., introduced the concept of fuzzy HX ring. In this paper we define a new algebraic structure of an intuitionistic fuzzy semi HX ring of a semi HX ring and investigate some related properties. We define the necessity and possibility operators of an intuitionistic fuzzy subset of an intuitionistic fuzzy semi HX ring and discuss some of its properties. Also we introduce the image and pre-image of an intuitionistic fuzzy set in an intuitionistic fuzzy semi HX ring and discuss some of its properties.

\section{PReliminaries}

In this section, we site the fundamental definitions that will be used in the sequel. Throughout this paper, $\mathrm{R}=(\mathrm{R},+, \cdot)$ is a semi Ring, $\mathrm{e}$ is the additive identity element of $\mathrm{R}$ and $\mathrm{xy}$, we mean $\mathrm{x} . \mathrm{y}$

\subsection{Definition}

Let $\mathrm{R}$ be a semi ring. Let $\mu$ be a fuzzy ring defined on R. Let $\vartheta \subset 2^{R}-\{\phi\}$ be a semi HX ring. A fuzzy subset $\lambda^{\mu}$ of $\vartheta$ is called a fuzzy semi HX ring on $\vartheta$ or a fuzzy semi ring induced by $\mu$ if the following conditions are satisfied. For all $\mathrm{A}, \mathrm{B} \in \vartheta$,

$$
\begin{array}{ll}
\text { i. } & \lambda^{\mu}(A+B) \geq \min \left\{\lambda^{\mu}(A), \lambda^{\mu}(B)\right\}, \\
\text { ii. } & \lambda^{\mu}(A B) \geq \min \left\{\lambda^{\mu}(A), \lambda^{\mu}(B)\right\}
\end{array}
$$

where $\lambda^{\mu}(\mathrm{A})=\max \{\mu(\mathrm{x}) /$ for all $\mathrm{x} \in \mathrm{A} \subseteq \mathrm{R}\}$. 


\subsection{Definition}

Let $\mathrm{G}=\{\langle\mathrm{x}, \alpha(\mathrm{x}), \beta(\mathrm{x})\rangle / \mathrm{x} \in \mathrm{R}\}$ and $\mathrm{H}=\{\langle\mathrm{x}, \mu(\mathrm{x}), \eta(\mathrm{x})\rangle / \mathrm{x} \in \mathrm{R}\}$ be any two intuitionistic fuzzy sets defined on a semi ring R. Let $\Re \subset 2^{R}-\{\phi\}$ be a semi HX ring on R . Let $\quad \gamma^{G}=\{$ $\left.\left\langle\mathrm{A}, \gamma^{\alpha}(\mathrm{A}), \gamma^{\beta}(\mathrm{A})\right\rangle / \mathrm{A} \in \mathfrak{R}\right\}$ and $\lambda^{\mathrm{H}}=\left\{\left\langle\mathrm{A}, \lambda^{\mu}(\mathrm{A}), \lambda^{\eta}(\mathrm{A})\right\rangle / \mathrm{A} \in \mathfrak{R}\right\}$ be any two intuitionistic fuzzy subsets of $\Re$. The intersection of $\gamma^{\mathrm{G}}$ and $\lambda^{\mathrm{H}}$ is defined as $\gamma^{\mathrm{G}} \cap \lambda^{\mathrm{H}}=\left\{\left\langle\mathrm{A},\left(\gamma^{\alpha} \cap \lambda^{\mu}\right)(\mathrm{A}),\left(\gamma^{\beta} \cup \lambda^{\eta}\right)(\mathrm{A})\right\rangle /\right.$ $\mathrm{A} \in \mathfrak{R}\}$

\subsection{Theorem}

Let $\mathrm{G}$ and $\mathrm{H}$ be any two intuitionistic fuzzy sets on R. Let $\gamma^{\mathrm{G}}$ and $\lambda^{\mathrm{H}}$ be any two intuitionistic fuzzy semi HX ring of a HX ring $\Re$ then their intersection, $\gamma^{\mathrm{G}} \cap \lambda^{\mathrm{H}}$ is also an intuitionistic fuzzy semi HX ring of a semi HX ring $\Re$.

\section{Proof}

Let $\mathrm{G}=\{\langle\mathrm{x}, \alpha(\mathrm{x}), \beta(\mathrm{x})\rangle / \mathrm{x} \in \mathrm{R}\}$ and $\mathrm{H}=\{\langle\mathrm{x}, \mu(\mathrm{x}), \eta(\mathrm{x})\rangle / \mathrm{x} \in \mathrm{R}\}$ be any two intuitionistic fuzzy sets defined on a semi ring $\mathrm{R}$.

Then, $\gamma^{\mathrm{G}}=\left\{\left\langle\mathrm{A}, \gamma^{\alpha}(\mathrm{A}), \gamma^{\beta}(\mathrm{A})\right\rangle / \mathrm{A} \in \mathfrak{R}\right\}$ and $\lambda^{\mathrm{H}}=\left\{\left\langle\mathrm{A}, \lambda^{\mu}(\mathrm{A}), \lambda^{\eta}(\mathrm{A})\right\rangle / \mathrm{A} \in \mathfrak{R}\right\}$ be any two intuitionistic fuzzy semi HX rings of a semi HX ring $\mathfrak{R}$. Then,

$$
\gamma^{\mathrm{G}} \cap \lambda^{\mathrm{H}}=\left\{\left\langle\mathrm{A},\left(\gamma^{\alpha} \cap \lambda^{\mu}\right)(\mathrm{A}),\left(\gamma^{\beta} \cup \lambda^{\eta}\right)(\mathrm{A})\right\rangle / \mathrm{A} \in \mathfrak{R}\right\}
$$

Let $\mathrm{A}, \mathrm{B} \in \mathfrak{R}$

\begin{tabular}{|c|c|c|c|}
\hline \multirow[t]{5}{*}{ i. } & $\left(\gamma^{\alpha} \cap \lambda^{\mu}\right)(\mathrm{A}+\mathrm{B})$ & $=$ & $\min \left\{\gamma^{\alpha}(\mathrm{A}+\mathrm{B}), \lambda^{\mu}(\mathrm{A}+\mathrm{B})\right\}$ \\
\hline & & $\geq$ & $\min \left\{\min \left\{\gamma^{\alpha}(\mathrm{A}), \gamma^{\alpha}(\mathrm{B})\right\}, \min \left\{\lambda^{\mu}(\mathrm{A}), \lambda^{\mu}(\mathrm{B})\right\}\right\}$ \\
\hline & & $=$ & $\min \left\{\min \left\{\gamma^{\alpha}(\mathrm{A}), \lambda^{\mu}(\mathrm{A})\right\}, \min \left\{\gamma^{\alpha}(\mathrm{B}), \lambda^{\mu}(\mathrm{B})\right\}\right\}$ \\
\hline & & $=$ & $\min \left\{\left(\gamma^{\alpha} \cap \lambda^{\mu}\right)(\mathrm{A}),\left(\gamma^{\alpha} \cap \lambda^{\mu}\right)(\mathrm{B})\right\}$ \\
\hline & $\left(\gamma^{\alpha} \cap \lambda^{\mu}\right)(\mathrm{A}+\mathrm{B})$ & $\geq$ & $\min \left\{\left(\gamma^{\alpha} \cap \lambda^{\mu}\right)(\mathrm{A}),\left(\gamma^{\alpha} \cap \lambda \lambda^{\mu}\right)(\mathrm{B})\right\}$ \\
\hline \multirow[t]{5}{*}{ ii. } & $\left(\gamma^{\alpha} \cap \lambda^{\mu}\right)(\mathrm{AB})$ & $=$ & $\min \left\{\gamma^{\alpha}(\mathrm{AB}), \lambda^{\mu}(\mathrm{AB})\right\}$ \\
\hline & & $\geq$ & $\min \left\{\min \left\{\gamma^{\alpha}(\mathrm{A}), \gamma^{\alpha}(\mathrm{B})\right\}, \min \left\{\lambda^{\mu}(\mathrm{A}), \lambda^{\mu}(\mathrm{B})\right\}\right\}$ \\
\hline & & $=$ & $\min \left\{\min \left\{\gamma^{\alpha}(\mathrm{A}), \lambda^{\mu}(\mathrm{A})\right\}, \min \left\{\gamma^{\alpha}(\mathrm{B}), \lambda^{\mu}(\mathrm{B})\right\}\right\}$ \\
\hline & & $=$ & $\min \left\{\left(\gamma^{\alpha} \cap \lambda^{\mu}\right)(\mathrm{A}),\left(\gamma^{\alpha} \cap \lambda^{\mu}\right)(\mathrm{B})\right\}$ \\
\hline & $\left(\gamma^{\alpha} \cap \lambda^{\mu}\right)(\mathrm{AB})$ & $\geq$ & $\min \left\{\left(\gamma^{\alpha} \cap \lambda^{\mu}\right)(\mathrm{A}),\left(\gamma^{\alpha} \cap \lambda^{\mu}\right)(\mathrm{B})\right\}$ \\
\hline \multirow[t]{5}{*}{ iii } & $\left(\gamma^{\beta} \cup \lambda^{\eta}\right)(\mathrm{A}+\mathrm{B})$ & $=$ & $\max \left\{\gamma^{\beta}(A+B), \lambda^{\eta}(A+B)\right\}$ \\
\hline & & $\leq$ & $\max \left\{\max \left\{\gamma^{\beta}(\mathrm{A}), \gamma^{\beta}(\mathrm{B})\right\}, \max \left\{\lambda^{\eta}(\mathrm{A}), \lambda^{\eta}(\mathrm{B})\right\}\right\}$ \\
\hline & & $=$ & $\max \left\{\max \left\{\gamma^{\beta}(\mathrm{A}), \lambda^{\eta}(\mathrm{A})\right\}, \max \left\{\gamma^{\beta}(\mathrm{B}), \lambda^{\eta}(\mathrm{B})\right\}\right\}$ \\
\hline & & $=$ & $\max \left\{\left(\gamma^{\beta} \cup \lambda^{\eta}\right)(\mathrm{A}),\left(\gamma^{\beta} \cup \lambda^{\eta}\right)(\mathrm{B})\right\}$ \\
\hline & $\left(\gamma^{\beta} \cup \lambda^{\mu}\right)(\mathrm{A}+\mathrm{B})$ & $\leq$ & $\max \left\{\left(\gamma^{\beta} \cup \gamma^{\eta}\right)(\mathrm{A}),\left(\gamma^{\beta} \cup \lambda^{\eta}\right)(\mathrm{B})\right\}$. \\
\hline \multirow[t]{5}{*}{ iv. } & $\left(\gamma^{\beta} \cup \lambda^{\eta}\right)(\mathrm{AB})$ & $=$ & $\max \left\{\gamma^{\beta}(\mathrm{AB}), \lambda^{\eta}(\mathrm{AB})\right\}$ \\
\hline & & $\leq$ & $\max \left\{\max \left\{\gamma^{\beta}(\mathrm{A}), \gamma^{\beta}(\mathrm{B})\right\}, \max \left\{\lambda^{\eta}(\mathrm{A}), \lambda^{\eta}(\mathrm{B})\right\}\right\}$ \\
\hline & & $=$ & $\max \left\{\max \left\{\gamma^{\beta}(\mathrm{A}), \lambda^{\eta}(\mathrm{A})\right\}, \max \left\{\gamma^{\beta}(\mathrm{B}), \lambda^{\eta}(\mathrm{B})\right\}\right\}$ \\
\hline & & $=$ & $\max \left\{\left(\gamma^{\beta} \cup \lambda^{\eta}\right)(\mathrm{A}),\left(\gamma^{\beta} \cup \lambda^{\eta}\right)(\mathrm{B})\right\}$ \\
\hline & & $\leq$ & $\max \left\{\left(\gamma^{\rho} \cup \gamma^{\prime \mid}\right)(\mathrm{A})\right.$ \\
\hline
\end{tabular}

Hence, $\gamma^{\mathrm{G}} \cap \lambda^{\mathrm{H}}$ is an intuitionistic fuzzy semi HX ring of a semi HX ring $\mathfrak{R}$.

\subsection{Theorem}

If $\gamma^{\mathrm{G}}, \lambda^{\mathrm{H}}, \varphi^{\mathrm{G} \cap \mathrm{H}}$ are intuitionistic fuzzy semi HX rings of a semi HX ring $\Re$ induced by intuitionistic fuzzy sets $G, H, G \cap H$ of $R$ then $\varphi^{\mathrm{G} \cap \mathrm{H}}=\gamma^{\mathrm{G}} \cap \lambda^{\mathrm{H}}$.

\section{Proof}

Let $\mathrm{G}=\{\langle\mathrm{x}, \alpha(\mathrm{x}), \beta(\mathrm{x})\rangle / \mathrm{x} \in \mathrm{R}\}$ and $\mathrm{H}=\{\langle\mathrm{x}, \mu(\mathrm{x}), \eta(\mathrm{x})\rangle / \mathrm{x} \in \mathrm{R}\}$ be any two intuitionistic fuzzy sets defined on a semi ring R. $G \cap H=\{\langle x,(\alpha \cap \mu)(x),(\beta \cup \eta)(x)\rangle / x \in R\}$.

Then, $\gamma^{\mathrm{G}}=\left\{\left\langle\mathrm{A}, \gamma^{\alpha}(\mathrm{A}), \gamma^{\beta}(\mathrm{A})\right\rangle / \mathrm{A} \in \mathfrak{R}\right\}$ and $\lambda^{\mathrm{H}}=\left\{\left\langle\mathrm{A}, \lambda^{\mu}(\mathrm{A}), \lambda^{\eta}(\mathrm{A})\right\rangle / \mathrm{A} \in \mathfrak{R}\right\}$ be any two intuitionistic fuzzy semi HX rings of a semi HX ring $\mathfrak{R}$.

$\gamma^{\mathrm{G}} \cap \lambda^{\mathrm{H}}=\left\{\left\langle\mathrm{A},\left(\gamma^{\alpha} \cap \lambda^{\mu}\right)(\mathrm{A}),\left(\gamma^{\beta} \cup \lambda^{\eta}\right)(\mathrm{A})\right\rangle / \mathrm{A} \in \mathfrak{R}\right\}$. By Theorem 2.1, $\gamma^{\mathrm{G}} \cap \lambda^{\mathrm{H}}$ is an intuitionistic fuzzy HX subring of a HX ring $\Re$. 
$\overline{\varphi^{\mathrm{G} \cap \mathrm{H}}}=\left\{\left\langle\mathrm{A}, \varphi^{\alpha \cap \mu}(\mathrm{A}), \varphi^{\beta \cup \eta}(\mathrm{A})\right\rangle / \mathrm{A} \in \mathfrak{R}\right\}$ and $\varphi^{\mathrm{G} \cap \mathrm{H}}$ is an intuitionistic fuzzy semi HX ring of $\mathfrak{R}$ induced by $\mathrm{G} \cap \mathrm{H}$ of $\mathrm{R}$.

$\begin{array}{rlll}\varphi^{\alpha \cap \mu}(\mathrm{A}) & = & \max \{(\alpha \cap \mu)(\mathrm{x}) / \text { for all } \mathrm{x} \in \mathrm{A} \subseteq \mathrm{R}\} \\ & = & \max \{\min \{\alpha(\mathrm{x}), \mu(\mathrm{x})\} / \text { for all } \mathrm{x} \in \mathrm{A} \subseteq \mathrm{R}\} \\ & = & \min \{\max \{\alpha(\mathrm{x}) / \text { for all } \mathrm{x} \in \mathrm{A} \subseteq \mathrm{R}\}, \max \{\mu(\mathrm{x}) / \text { for all } \mathrm{x} \in \mathrm{A} \subseteq \mathrm{R}\}\} \\ & = & \min \left\{\gamma^{\alpha}(\mathrm{A}), \lambda^{\mu}(\mathrm{A})\right\} \\ \varphi^{\alpha \cap \mu}(\mathrm{A}) & = & \left(\gamma^{\alpha} \cap \lambda^{\mu}\right)(\mathrm{A}), \text { for any } \mathrm{A} \in \mathfrak{R} . \\ \text { Hence, } \varphi^{\alpha \cap \mu} & = & \gamma^{\alpha} \cap \lambda^{\mu} . \\ \text { Similarly, } \varphi^{\beta \cup \eta}(\mathrm{A}) & = & \min \{(\beta \cup \eta)(\mathrm{x}) / \text { for all } \mathrm{x} \in \mathrm{A} \subseteq \mathrm{R}\} \\ & = & \min \{\max \{\beta(\mathrm{x}), \eta(\mathrm{x})\} / \text { for all } \mathrm{x} \in \mathrm{A} \subseteq \mathrm{R}\} \\ & = & \max \{\min \{\beta(\mathrm{x}) / \text { for all } \mathrm{x} \in \mathrm{A} \subseteq \mathrm{R}\}, \min \{\eta(\mathrm{x}) / \text { for all } \mathrm{x} \in \mathrm{A} \subseteq \mathrm{R}\}\} \\ & & & \max \left\{\gamma^{\beta}(\mathrm{A}), \lambda^{\eta}(\mathrm{A})\right\} \\ \text { Hence, } \varphi^{\beta \cup \eta} & = & \left(\gamma^{\beta} \cup \lambda^{\eta}\right)(\mathrm{A}), \text { for any } \mathrm{A} \in \mathfrak{R} . \\ \text { Hence, } \varphi^{\mathrm{G} \cap \mathrm{H}} & = & \gamma^{\beta} \cup \lambda^{\eta} .\end{array}$

\subsection{Definition}

Let $\mathrm{G}=\{\langle\mathrm{x}, \alpha(\mathrm{x}), \beta(\mathrm{x})\rangle / \mathrm{x} \in \mathrm{R}\}$ and $\mathrm{H}=\{\langle\mathrm{x}, \mu(\mathrm{x}), \eta(\mathrm{x})\rangle / \mathrm{x} \in \mathrm{R}\}$ be any two intuitionistic fuzzy sets defined on a semi ring $\mathrm{R}$. Let $\mathfrak{R} \subset 2^{\mathrm{R}}-\{\phi\}$ be a semi $\mathrm{HX}$ ring of $\mathrm{R}$. Let $\gamma^{\mathrm{G}}=\left\{\left\langle\mathrm{A}, \gamma^{\alpha}(\mathrm{A}), \gamma^{\beta}(\mathrm{A})\right\rangle / \mathrm{A} \in \mathfrak{R}\right\}$ and $\lambda^{\mathrm{H}}=\left\{\left\langle\mathrm{A}, \lambda^{\mu}(\mathrm{A}), \lambda^{\eta}(\mathrm{A})\right\rangle / \mathrm{A} \in \mathfrak{R}\right\}$ be any two intuitionistic fuzzy subsets of a semi $\mathrm{HX}$ ring $\mathfrak{R}$. The union of $\gamma^{\mathrm{G}}$ and $\lambda^{\mathrm{H}}$ is defined as $\gamma^{\mathrm{G}} \cup \lambda^{\mathrm{H}}=\left\{\left\langle\mathrm{A},\left(\gamma^{\alpha} \cup \lambda^{\mu}\right)(\mathrm{A}),\left(\gamma^{\beta} \cap \lambda^{\eta}\right)(\mathrm{A})\right\rangle / \mathrm{A} \in \mathfrak{R}\right\}$.

\subsection{Theorem}

Let $\mathrm{G}$ and $\mathrm{H}$ be any two intuitionistic fuzzy sets on R. Let $\gamma^{\mathrm{G}}$ and $\lambda^{\mathrm{H}}$ be any two intuitionistic fuzzy semi HX rings of a semi HX ring $\Re$ then their union, $\gamma^{\mathrm{G}} \cup \lambda^{\mathrm{H}}$ is also an intuitionistic fuzzy semi HX ring of a semi HX ring $\Re$.

\section{Proof}

Let $\mathrm{G}=\{\langle\mathrm{x}, \alpha(\mathrm{x}), \beta(\mathrm{x})\rangle / \mathrm{x} \in \mathrm{R}\}$ and $\mathrm{H}=\{\langle\mathrm{x}, \mu(\mathrm{x}), \eta(\mathrm{x})\rangle / \mathrm{x} \in \mathrm{R}\}$ be any two intuitionistic fuzzy sets defined on a semi ring $\mathrm{R}$.

Then, $\gamma^{\mathrm{G}}=\left\{\left\langle\mathrm{A}, \gamma^{\alpha}(\mathrm{A}), \gamma^{\beta}(\mathrm{A})\right\rangle / \mathrm{A} \in \mathfrak{R}\right\}$ and $\lambda^{\mathrm{H}}=\left\{\left\langle\mathrm{A}, \lambda^{\mu}(\mathrm{A}), \lambda^{\eta}(\mathrm{A})\right\rangle / \mathrm{A} \in \mathfrak{R}\right\}$ be any two intuitionistic fuzzy semi HX ring of a semi HX ring R.Then,

$$
\gamma^{\mathrm{G}} \cup \lambda^{\mathrm{H}}=\left\{\left\langle\mathrm{A},\left(\gamma^{\alpha} \cup \lambda^{\mu}\right)(\mathrm{A}),\left(\gamma^{\beta} \cap \lambda^{\eta}\right)(\mathrm{A})\right\rangle / \mathrm{A} \in \mathfrak{R}\right\}
$$

Let $\mathrm{A}, \mathrm{B} \in \mathfrak{R}$

\begin{tabular}{|c|c|c|c|}
\hline \multirow[t]{5}{*}{ i. } & $\left(\gamma^{\alpha} \cup \lambda^{\mu}\right)(\mathrm{A}+\mathrm{B})$ & $=$ & $\max \left\{\gamma^{\alpha}(\mathrm{A}+\mathrm{B}), \lambda^{\mu}(\mathrm{A}+\mathrm{B})\right\}$ \\
\hline & & $\geq$ & $\max \left\{\min \left\{\gamma^{\alpha}(\mathrm{A}), \gamma^{\alpha}(\mathrm{B})\right\}, \min \left\{\lambda^{\mu}(\mathrm{A}), \lambda^{\mu}(\mathrm{B})\right\}\right\}$ \\
\hline & & $=$ & $\min \left\{\max \left\{\gamma^{\alpha}(\mathrm{A}), \lambda^{\mu}(\mathrm{A})\right\}, \max \left\{\gamma^{\alpha}(\mathrm{B}), \lambda^{\mu}(\mathrm{B})\right\}\right\}$ \\
\hline & & $=$ & $\min \left\{\left(\gamma^{\alpha} \cup \lambda^{\mu}\right)(\mathrm{A}),\left(\gamma^{\alpha} \cup \lambda^{\mu}\right)(\mathrm{B})\right\}$ \\
\hline & $\left(\gamma^{\alpha} \cup \lambda^{\mu}\right)(\mathrm{A}+\mathrm{B})$ & $\geq$ & $\min \left\{\left(\gamma^{\alpha} \cup \lambda^{\mu}\right)(\mathrm{A}),\left(\gamma^{\alpha} \cup \lambda^{\mu}\right)(\mathrm{B})\right\}$ \\
\hline \multirow[t]{5}{*}{ ii. } & $\left(\gamma^{\alpha} \cup \gamma^{\mu}\right)(\mathrm{AB})$ & $=$ & $\max \left\{\gamma^{\alpha}(\mathrm{AB}), \lambda^{\mu}(\mathrm{AB})\right\}$ \\
\hline & & $\geq$ & $\max \left\{\min \left\{\gamma^{\alpha}(\mathrm{A}), \gamma^{\alpha}(\mathrm{B})\right\}, \min \left\{\lambda^{\mu}(\mathrm{A}), \lambda^{\mu}(\mathrm{B})\right\}\right\}$ \\
\hline & & $=$ & $\min \left\{\max \left\{\gamma^{\alpha}(\mathrm{A}), \lambda^{\mu}(\mathrm{A})\right\}, \max \left\{\gamma^{\alpha}(\mathrm{B}), \lambda^{\mu}(\mathrm{B})\right\}\right\}$ \\
\hline & & $=$ & $\min \left\{\left(\gamma^{\alpha} \cup \lambda^{\mu}\right)(\mathrm{A}),\left(\gamma^{\alpha} \cup \lambda^{\mu}\right)(\mathrm{B})\right\}$ \\
\hline & $\left(\gamma^{\alpha} \cup \lambda^{\mu}\right)(\mathrm{AB})$ & $\geq$ & $\min \left\{\left(\gamma^{\alpha} \cup \lambda^{\mu}\right)(\mathrm{A}),\left(\gamma^{\alpha} \cup \lambda^{\mu}\right)(\mathrm{B})\right\}$ \\
\hline \multirow[t]{5}{*}{ iii. } & $\left(\gamma^{\beta} \cap \lambda^{\eta}\right)(A+B)$ & $=$ & $\min \left\{\gamma^{\beta}(A+B), \lambda^{\eta}(A+B)\right\}$ \\
\hline & & $\leq$ & $\min \left\{\max \left\{\gamma^{\beta}(\mathrm{A}), \gamma^{\beta}(\mathrm{B})\right\}, \max \left\{\lambda^{\eta}(\mathrm{A}), \lambda^{\eta}(\mathrm{B})\right\}\right\}$ \\
\hline & & $=$ & $\max \left\{\min \left\{\gamma^{\beta}(\mathrm{A}), \lambda^{\eta}(\mathrm{A})\right\}, \min \left\{\gamma^{\beta}(\mathrm{B}), \lambda^{\eta}(\mathrm{B})\right\}\right\}$ \\
\hline & & $=$ & $\max \left\{\left(\gamma^{\beta} \cap \gamma^{\eta}\right)(\mathrm{A}),\left(\gamma^{\beta} \cup \lambda^{\eta}\right)(\mathrm{B})\right\}$ \\
\hline & $\left(\gamma^{\beta} \cap \lambda^{\mu}\right)(A+B)$ & $\leq$ & $\max \left\{\left(\gamma^{\beta} \cap \gamma^{\eta}\right)(\mathrm{A}),\left(\gamma^{\beta} \cap \lambda^{\eta}\right)(\mathrm{B})\right\}$ \\
\hline \multirow[t]{2}{*}{ iv. } & $\left(\gamma^{\beta} \cap \lambda^{\eta}\right)(\mathrm{AB})$ & $=$ & $\min \left\{\gamma^{\beta}(\mathrm{AB}), \lambda^{\eta}(\mathrm{AB})\right\}$ \\
\hline & & $\leq$ & $\min \left\{\max \left\{\gamma^{\beta}(\mathrm{A}), \gamma^{\beta}(\mathrm{B})\right\}, \max \left\{\lambda^{\eta}(\mathrm{A}), \lambda^{\eta}(\mathrm{B})\right\}\right\}$ \\
\hline
\end{tabular}




$$
\begin{array}{rll} 
& = & \max \left\{\min \left\{\gamma^{\beta}(\mathrm{A}), \lambda^{\eta}(\mathrm{A})\right\}, \min \left\{\gamma^{\beta}(\mathrm{B}), \lambda^{\eta}(\mathrm{B})\right\}\right\} \\
& = & \max \left\{\left(\gamma^{\beta} \cap \gamma^{\eta}\right)(\mathrm{A}),\left(\gamma^{\beta} \cup \lambda^{\eta}\right)(\mathrm{B})\right\} \\
\left(\gamma^{\beta} \cap \lambda^{\mu}\right)(\mathrm{AB}) & \leq & \max \left\{\left(\gamma^{\beta} \cap \gamma^{\eta}\right)(\mathrm{A}),\left(\gamma^{\beta} \cap \lambda^{\eta}\right)(\mathrm{B})\right\} .
\end{array}
$$

Hence, $\gamma^{\mathrm{G}} \cup \lambda^{\mathrm{H}}$ is an intuitionistic fuzzy semi HX ring of a semi HX ring $\Re$.

\subsection{Theorem}

Let $\mathrm{R}$ be a semi ring. Let $\mathrm{G}$ and $\mathrm{H}$ be any two intuitionistic fuzzy sets of $\mathrm{R}$. If $\gamma^{\mathrm{G}}$, $\lambda^{\mathrm{H}}$, $\varphi^{\mathrm{G} \cup \mathrm{H}}$ are intuitionistic fuzzy semi HX rings of a semi HX ring $\mathfrak{R}$ induced by intuitionistic fuzzy sets $\mathrm{G}, \mathrm{H}, \mathrm{G} \cup \mathrm{H}$ of R then $\varphi^{\mathrm{G} \cup \mathrm{H}}=\gamma^{\mathrm{G}} \cup \lambda^{\mathrm{H}}$.

\section{Proof}

It is clear.

\subsection{Definition}

Let $\mathrm{G}=\{\langle\mathrm{x}, \alpha(\mathrm{x}), \beta(\mathrm{x})\rangle / \mathrm{x} \in \mathrm{R}\}$ and $\mathrm{H}=\{\langle\mathrm{x}, \mu(\mathrm{x}), \eta(\mathrm{x})\rangle / \mathrm{x} \in \mathrm{R}\}$ be any two intuitionistic fuzzy sets defined on a semi ring R. . Let $\mathfrak{R}_{1} \subset 2^{\mathrm{R}}{ }_{1}-\{\phi\}$ and $\mathfrak{R}_{2} \subset 2^{\mathrm{R}}{ }_{2}-\{\phi\}$ be any two semi HX rings. Let $\gamma^{\mathrm{G}}=\left\{\left\langle\mathrm{A}, \gamma^{\alpha}(\mathrm{A}), \gamma^{\beta}(\mathrm{A})\right\rangle / \mathrm{A} \in \mathfrak{R}\right\}$ and $\lambda^{\mathrm{H}}=\left\{\left\langle\mathrm{A}, \lambda^{\mu}(\mathrm{A}), \lambda^{\eta}(\mathrm{A})\right\rangle / \mathrm{A} \in \mathfrak{R}\right\}$ be any two intuitionistic fuzzy subsets of a semi HX ring $\Re$, then the cartesian product of $\gamma^{\mathrm{G}}$ and $\lambda^{\mathrm{H}}$ is defined as $\left(\gamma^{\mathrm{G}} \times \lambda^{\mathrm{H}}\right)=\left\{\left\langle(\mathrm{A}, \mathrm{B}),\left(\gamma^{\alpha} \cap \lambda^{\mu}\right)(\mathrm{A}, \mathrm{B}),\left(\gamma^{\beta} \cup \lambda^{\eta}\right)(\mathrm{A}, \mathrm{B})\right\rangle /(\mathrm{A}, \mathrm{B}) \in \mathfrak{R}_{1} \times \mathfrak{R}_{2}\right\}$,

where

$$
\begin{array}{ll}
\left(\gamma^{\alpha} \cap \lambda^{\mu}\right)(\mathrm{A}, \mathrm{B})= & \min \left\{\gamma^{\alpha}(\mathrm{A}), \lambda^{\mu}(\mathrm{B})\right\}, \text { for all }(\mathrm{A}, \mathrm{B}) \in \mathfrak{R}_{1} \times \mathfrak{R}_{2}, \\
\left(\gamma^{\beta} \cup \lambda^{\eta}\right)(\mathrm{A}, \mathrm{B})= & \max \left\{\gamma^{\beta}(\mathrm{A}), \lambda^{\eta}(\mathrm{B})\right\}, \text { for all }(\mathrm{A}, \mathrm{B}) \in \mathfrak{R}_{1} \times \mathfrak{R}_{2} .
\end{array}
$$

\subsection{Theorem}

Let $G$ and $H$ be any two intuitionistic fuzzy sets of $R_{1}$ and $R_{2}$ respectively. Let $\Re_{1} \subset 2^{R}{ }_{1}-\{\phi\}$ and $\Re_{2} \subset 2^{\mathrm{R}}{ }_{2}-\{\phi\}$ be any two semi HX rings. If $\gamma^{\mathrm{G}}$ and $\lambda^{\mathrm{H}}$ are any two intuitionistic fuzzy semi HX rings of $\Re_{1}$ and $\Re_{2}$ respectively then, $\gamma^{\mathrm{G}} \times \lambda^{\mathrm{H}}$ is also an intuitionistic fuzzy semi HX ring of a semi HX ring $\Re_{1} \times \Re_{2}$.

\section{Proof}

Let $\mathrm{G}=\left\{\langle\mathrm{x}, \alpha(\mathrm{x}), \beta(\mathrm{x})\rangle / \mathrm{x} \in \mathrm{R}_{1}\right\}$ and $\mathrm{H}=\left\{\langle\mathrm{x}, \mu(\mathrm{x}), \eta(\mathrm{x})\rangle / \mathrm{x} \in \mathrm{R}_{2}\right\}$ be any two intuitionistic fuzzy sets defined on a semi ring $R_{1}$ and $R_{2}$ respectively.

Then, $\gamma^{\mathrm{G}}=\left\{\left\langle\mathrm{A}, \gamma^{\alpha}(\mathrm{A}), \gamma^{\beta}(\mathrm{A})\right\rangle / \mathrm{A} \in \mathfrak{R}_{1}\right\}$ and $\lambda^{\mathrm{H}}=\left\{\left\langle\mathrm{A}, \lambda^{\mu}(\mathrm{A}), \lambda^{\eta}(\mathrm{A})\right\rangle / \mathrm{A} \in \mathfrak{R}_{2}\right\}$ be any two intuitionistic fuzzy semi HX ring of a semi HX ring $\mathfrak{R}_{1}$ and $\mathfrak{R}_{2}$ respectively. Then,

$\left(\gamma^{\mathrm{G}} \times \lambda^{\mathrm{H}}\right)=\left\{\left\langle(\mathrm{A}, \mathrm{B}),\left(\gamma^{\alpha} \cap \lambda^{\mu}\right)(\mathrm{A}, \mathrm{B}),\left(\gamma^{\beta} \cup \lambda^{\eta}\right)(\mathrm{A}, \mathrm{B})\right\rangle /(\mathrm{A}, \mathrm{B}) \in \mathfrak{R}_{1} \times \mathfrak{R}_{2}\right\}$,

where $\quad\left(\gamma^{\alpha} \cap \lambda^{\mu}\right)(\mathrm{A}, \mathrm{B})=\quad \min \left\{\gamma^{\alpha}(\mathrm{A}), \lambda^{\mu}(\mathrm{B})\right\}$, for all $(\mathrm{A}, \mathrm{B}) \in \mathfrak{R}_{1} \times \mathfrak{R}_{2}$,

$$
\left(\gamma^{\beta} \cup \lambda^{\eta}\right)(\mathrm{A}, \mathrm{B})=\quad \max \left\{\gamma^{\beta}(\mathrm{A}), \lambda^{\eta}(\mathrm{B})\right\}, \text { for all }(\mathrm{A}, \mathrm{B}) \in \mathfrak{R}_{1} \times \mathfrak{R}_{2} .
$$

Let $\mathrm{A}, \mathrm{B} \in \mathfrak{R}_{1} \times \mathfrak{R}_{2}$, where $\mathrm{A}=(\mathrm{C}, \mathrm{D}), \mathrm{B}=(\mathrm{E}, \mathrm{F})$,

i. $\quad \begin{array}{rlll}\quad\left(\gamma^{\alpha} \cap \lambda^{\mu}\right)(\mathrm{A}+\mathrm{B}) & & \min \left\{\gamma^{\alpha}(\mathrm{C}+\mathrm{E}), \lambda^{\mu}(\mathrm{D}+\mathrm{F})\right\} \\ & \geq & \min \left\{\min \left\{\gamma^{\alpha}(\mathrm{C}), \gamma^{\alpha}(\mathrm{E})\right\}, \min \left\{\lambda^{\mu}(\mathrm{D}), \lambda^{\mu}(\mathrm{F})\right\}\right\} \\ & = & \min \left\{\min \left\{\gamma^{\alpha}(\mathrm{C}), \lambda^{\mu}(\mathrm{D})\right\}, \min \left\{\gamma^{\alpha}(\mathrm{D}), \lambda^{\mu}(\mathrm{E})\right\}\right\} \\ & = & \min \left\{\left(\gamma^{\alpha} \cap \lambda^{\mu}\right)(\mathrm{C}, \mathrm{D}),\left(\gamma^{\alpha} \cap \lambda^{\mu}\right)(\mathrm{E}, \mathrm{F})\right\} \\ \text { ii. } \quad\left(\gamma^{\alpha} \cap \lambda^{\mu}\right)(\mathrm{A}+\mathrm{B}) & \geq & \min \left\{\left(\gamma^{\alpha} \cap \lambda^{\mu}\right)(\mathrm{A}),\left(\gamma^{\alpha} \cap \lambda^{\mu}\right)(\mathrm{B})\right\} . \\ & = & \left(\gamma^{\alpha} \cap \lambda^{\mu}\right)((\mathrm{C}, \mathrm{D}) \cdot(\mathrm{E}, \mathrm{F})) \\ & = & \left(\gamma^{\alpha} \cap \lambda^{\mu}\right)(\mathrm{CE}, \mathrm{DF}) \\ & = & \min \left\{\gamma^{\alpha}(\mathrm{CE}), \lambda^{\mu}(\mathrm{DF})\right\} \\ & \geq & \min \left\{\min \left\{\gamma^{\alpha}(\mathrm{C}), \gamma^{\alpha}(\mathrm{E})\right\}, \min \left\{\lambda^{\mu}(\mathrm{D}), \lambda^{\mu}(\mathrm{F})\right\}\right\} \\ & = & \min \left\{\min \left\{\gamma^{\alpha}(\mathrm{C}), \lambda^{\mu}(\mathrm{D})\right\}, \min \left\{\gamma^{\alpha}(\mathrm{E}), \lambda^{\mu}(\mathrm{F})\right\}\right\} \\ & =\min \left\{\left(\gamma^{\alpha} \cap \lambda^{\mu}\right)(\mathrm{C}, \mathrm{D}),\left(\gamma^{\alpha} \cap \lambda^{\mu}\right)(\mathrm{E}, \mathrm{F})\right\} \\ & \quad\left(\gamma^{\alpha} \cap \lambda^{\mu}\right)(\mathrm{AB}) & \geq & \min \left\{\left(\gamma^{\alpha} \cap \lambda^{\mu}\right)(\mathrm{A}),\left(\gamma^{\alpha} \cap \lambda^{\mu}\right)(\mathrm{B})\right\} . \\ \text { iii. } \quad\left(\gamma^{\beta} \cup \lambda^{\eta}\right)(\mathrm{A}+\mathrm{B}) \quad & = & \max \left\{\gamma^{\beta}(\mathrm{C}+\mathrm{E}), \lambda^{\eta}(\mathrm{D}+\mathrm{F})\right\}\end{array}$




$\begin{array}{rll} & \leq & \max \left\{\max \left\{\gamma^{\beta}(\mathrm{C}), \gamma^{\beta}(\mathrm{E})\right\}, \max \left\{\lambda^{\eta}(\mathrm{D}), \lambda^{\eta}(\mathrm{F})\right\}\right\} \\ & = & \max \left\{\max \left\{\gamma^{\beta}(\mathrm{C}), \lambda^{\eta}(\mathrm{D})\right\}, \max \left\{\gamma^{\beta}(\mathrm{E}), \lambda^{\eta}(\mathrm{F})\right\}\right\} \\ & = & \max \left\{\left(\gamma^{\beta} \cup \lambda^{\eta}\right)(\mathrm{C}, \mathrm{D}),\left(\gamma^{\beta} \cup \lambda^{\eta}\right)(\mathrm{E}, \mathrm{F})\right\} \\ \text { iv. } \quad\left(\gamma^{\beta} \cup \lambda^{\eta}\right)(\mathrm{A}+\mathrm{B}) & \leq & \max \left\{\left(\gamma^{\beta} \cup \lambda^{\eta}\right)(\mathrm{A}),\left(\gamma^{\beta} \cup \lambda^{\eta}\right)(\mathrm{B})\right\} . \\ & = & \left(\gamma^{\beta} \cup \lambda^{\eta}\right)((\mathrm{C}, \mathrm{D}) \cdot(\mathrm{E}, \mathrm{F})) \\ & = & \left(\gamma^{\beta} \cup \lambda^{\eta}\right)(\mathrm{CE}, \mathrm{DF}) \\ & = & \max \left\{\gamma^{\beta}(\mathrm{CE}), \lambda^{\eta}(\mathrm{DF})\right\} \\ & \leq & \max \left\{\max \left\{\gamma^{\beta}(\mathrm{C}), \gamma^{\beta}(\mathrm{E})\right\}, \max \left\{\lambda^{\eta}(\mathrm{D}), \lambda^{\eta}(\mathrm{F})\right\}\right\} \\ & = & \max \left\{\max \left\{\gamma^{\beta}(\mathrm{C}), \lambda^{\eta}(\mathrm{D})\right\}, \max \left\{\gamma^{\beta}(\mathrm{E}), \lambda^{\eta}(\mathrm{F})\right\}\right\} \\ & = & \max \left\{\left(\gamma^{\beta} \cup \lambda^{\eta}\right)(\mathrm{C}, \mathrm{D}),\left(\gamma^{\beta} \cup \lambda^{\eta}\right)(\mathrm{E}, \mathrm{F})\right\} \\ & \leq & \max \left\{\left(\gamma^{\beta} \cup \lambda^{\eta}\right)(\mathrm{A}),\left(\gamma^{\beta} \cup \lambda^{\eta}\right)(\mathrm{B})\right\} .\end{array}$

Hence, $\gamma^{\mathrm{G}} \times \lambda^{\mathrm{H}}$ is an intuitionistic fuzzy semi HX ring of a semi HX ring $\mathfrak{R}$.

\subsection{Theorem}

Let $\mathrm{G}$ and $\mathrm{H}$ be any two intuitionistic fuzzy sets of $\mathrm{R}_{1}$ and $\mathrm{R}_{2}$ respectively. Let $\Re_{1} \subset 2^{\mathrm{R}}{ }_{1}-\{\phi\}$ and $\Re_{2} \subset 2^{\mathrm{R}}{ }_{2}-\{\phi\}$ be any two semi HX rings. If $\gamma^{\mathrm{G}}$ and $\lambda^{\mathrm{H}}$ are any two intuitionistic fuzzy semi HX rings of $\mathfrak{R}_{1}$ and $\mathfrak{R}_{2}$ respectively. Suppose that $\mathrm{Q}$ and $\mathrm{Q}^{1}$ are identity elements of $\mathfrak{R}_{1}$ and $\mathfrak{R}_{2}$ respectively. If $\gamma^{\mathrm{G}} \times \lambda^{\mathrm{H}}$ is an intuitionistic fuzzy semi HX ring of $\mathfrak{R}_{1} \times \mathfrak{R}_{2}$, then atleast one of the following statements must hold

$$
\begin{array}{ll}
\text { i. } \lambda^{\mu}\left(\mathrm{Q}^{1}\right) & \geq \gamma^{\alpha}(\mathrm{A}) \text { and } \lambda^{\eta}\left(\mathrm{Q}^{1}\right) \leq \gamma^{\beta}(\mathrm{A}) \text {, for all } \mathrm{A} \in \mathfrak{R}_{1} \\
\text { ii. } \gamma^{\alpha}(\mathrm{Q}) & \geq \lambda^{\mu}(\mathrm{B}) \text { and } \gamma^{\beta}(\mathrm{Q}) \leq \lambda^{\eta}(\mathrm{B}), \text { for all } \mathrm{B} \in \mathfrak{R}_{2} .
\end{array}
$$

\section{Proof}

Let $\mathrm{G}=\left\{\langle\mathrm{x}, \alpha(\mathrm{x}), \beta(\mathrm{x})\rangle / \mathrm{x} \in \mathrm{R}_{1}\right\}$ and $\mathrm{H}=\left\{\langle\mathrm{x}, \mu(\mathrm{x}), \eta(\mathrm{x})\rangle / \mathrm{x} \in \mathrm{R}_{2}\right\}$ be any two intuitionistic fuzzy sets defined on a ring $\mathrm{R}_{1}$ and $\mathrm{R}_{2}$ respectively.

Then, $\gamma^{\mathrm{G}}=\left\{\left\langle\mathrm{A}, \gamma^{\alpha}(\mathrm{A}), \gamma^{\beta}(\mathrm{A})\right\rangle / \mathrm{A} \in \mathfrak{R}_{1}\right\}$ and $\lambda^{\mathrm{H}}=\left\{\left\langle\mathrm{A}, \lambda^{\mu}(\mathrm{A}), \lambda^{\eta}(\mathrm{A})\right\rangle / \mathrm{A} \in \mathfrak{R}_{2}\right\}$ be any two intuitionistic fuzzy semi HX ring of a semi HX ring $\mathfrak{R}_{1}$ and $\mathfrak{R}_{2}$ respectively. Then,

$\left(\gamma^{\mathrm{G}} \times \lambda^{\mathrm{H}}\right)=\left\{\left\langle(\mathrm{A}, \mathrm{B}),\left(\gamma^{\alpha} \cap \lambda^{\mu}\right)(\mathrm{A}, \mathrm{B}),\left(\gamma^{\beta} \cup \lambda^{\eta}\right)(\mathrm{A}, \mathrm{B})\right\rangle /(\mathrm{A}, \mathrm{B}) \in \mathfrak{R}_{1} \times \mathfrak{R}_{2}\right\}$,

where $\quad\left(\gamma^{\alpha} \cap \lambda^{\mu}\right)(\mathrm{A}, \mathrm{B})=\quad \min \left\{\gamma^{\alpha}(\mathrm{A}), \lambda^{\mu}(\mathrm{B})\right\}$, for all $(\mathrm{A}, \mathrm{B}) \in \mathfrak{R}_{1} \times \mathfrak{R}_{2}$,

$$
\left(\gamma^{\beta} \cup \lambda^{\eta}\right)(\mathrm{A}, \mathrm{B})=\max \left\{\gamma^{\beta}(\mathrm{A}), \lambda^{\eta}(\mathrm{B})\right\}, \text { for all }(\mathrm{A}, \mathrm{B}) \in \mathfrak{R}_{1} \times \mathfrak{R}_{2} .
$$

Let $\mathrm{A}, \mathrm{B} \in \mathfrak{R}_{1} \times \mathfrak{R}_{2}$, where $\mathrm{A}=(\mathrm{C}, \mathrm{D}), \mathrm{B}=(\mathrm{E}, \mathrm{F})$,

Let $\gamma^{\mathrm{G}} \times \lambda^{\mathrm{H}}$ be an intuitionistic fuzzy semi HX ring of $\Re_{1} \times \Re_{2}$. By contraposition, suppose that none of the statements (i) and (ii) holds then we can find $\mathrm{A} \in \mathfrak{R}_{1}$ and $\mathrm{B} \in \mathfrak{R}_{2}$ such that

$$
\begin{aligned}
\text { i. } \lambda^{\mu}\left(\mathrm{Q}^{1}\right) & \leq \gamma^{\alpha}(\mathrm{A}) \text { and } \lambda^{\eta}\left(\mathrm{Q}^{1}\right) \geq \gamma^{\beta}(\mathrm{A}), \text { for all } \mathrm{A} \in \mathfrak{R}_{1} \\
\text { ii. } \gamma^{\alpha}(\mathrm{Q}) & \leq \lambda^{\mu}(\mathrm{B}) \text { and } \gamma^{\beta}(\mathrm{Q}) \geq \lambda^{\eta}(\mathrm{A}) \text {, for all } \mathrm{B} \in \mathfrak{R}_{2} .
\end{aligned}
$$

We have,

Also,

$$
\begin{array}{rll}
\left(\gamma^{\alpha} \cap \lambda^{\mu}\right)(\mathrm{A}, \mathrm{B}) & = & \min \left\{\gamma^{\alpha}(\mathrm{A}), \lambda^{\mu}(\mathrm{B})\right\} \\
& > & \min \left\{\lambda^{\mu}\left(\mathrm{Q}^{1}\right), \gamma^{\alpha}(\mathrm{Q})\right\} \\
& = & \min \left\{\gamma^{\alpha}(\mathrm{Q}), \lambda^{\mu}\left(\mathrm{Q}^{1}\right)\right\} \\
& = & \left(\gamma^{\alpha} \cap \lambda^{\mu}\right)\left(\mathrm{Q}, \mathrm{Q}^{1}\right) \\
\left(\gamma^{\alpha} \cap \lambda^{\mu}\right)(\mathrm{A}, \mathrm{B}) & > & \left(\gamma^{\alpha} \cap \lambda^{\mu}\right)\left(\mathrm{Q}, \mathrm{Q}^{1}\right) . \\
\left(\gamma^{\beta} \cup \lambda^{\eta}\right)(\mathrm{A}, \mathrm{B}) & = & \max \left\{\gamma^{\beta}(\mathrm{A}), \lambda^{\eta}(\mathrm{B})\right\} \\
& < & \max \left\{\lambda^{\eta}\left(\mathrm{Q}^{1}\right), \gamma^{\beta}(\mathrm{Q})\right\} \\
& = & \max \left\{\gamma^{\beta}(\mathrm{Q}), \lambda^{\eta}\left(\mathrm{Q}^{1}\right)\right\} \\
\left(\gamma^{\beta} \cup \lambda^{\eta}\right)(\mathrm{A}, \mathrm{B}) & = & \left(\gamma^{\beta} \cup \lambda^{\eta}\right)\left(\mathrm{Q}, \mathrm{Q}^{1}\right) . \\
& < & \left(\gamma^{\beta} \cup \lambda^{\eta}\right)\left(\mathrm{Q}, \mathrm{Q}^{1}\right) .
\end{array}
$$

Thus, $\gamma^{\mathrm{G}} \times \lambda^{\mathrm{H}}$ is not an intuitionistic fuzzy semi HX ring of $\mathfrak{R}_{1} \times \mathfrak{R}_{2}$.

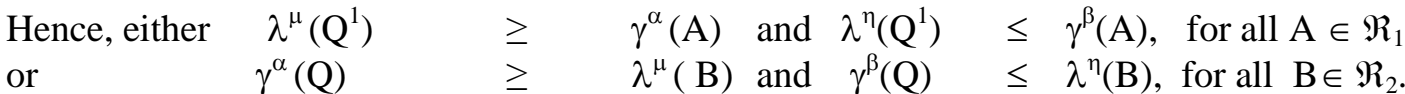




\subsection{Theorem}

Let $\mathrm{G}$ and $\mathrm{H}$ be any two intuitionistic fuzzy sets of $\mathrm{R}_{1}$ and $\mathrm{R}_{2}$ respectively. Let $\Re_{1} \subset 2^{\mathrm{R}}{ }_{1}-\{\phi\}$ and $\Re_{2} \subset 2^{\mathrm{R}}{ }_{2}-\{\phi\}$ be any two semi HX rings. If $\gamma^{\mathrm{G}}$ and $\lambda^{\mathrm{H}}$ are any two intuitionistic fuzzy semi HX rings of $\Re_{1}$ and $\Re_{2}$ respectively, such that $\lambda^{\mu}\left(Q^{1}\right) \geq \gamma^{\alpha}(A)$ and $\lambda^{\eta}\left(Q^{1}\right) \leq \gamma^{\beta}(A)$, for all $A \in \mathfrak{R}_{1}$, $Q^{1}$ being the identity element of $\mathfrak{R}_{2}$. If $\gamma^{\mathrm{G}} \times \lambda^{\mathrm{H}}$ is an intuitionistic fuzzy semi HX ring of $\mathfrak{R}_{1} \times \Re_{2}$, then $\gamma^{\mathrm{G}}$ is an intuitionistic fuzzy semi HX ring of $\Re_{1}$.

\section{Proof}

Let $\mathrm{G}=\left\{\langle\mathrm{x}, \alpha(\mathrm{x}), \beta(\mathrm{x})\rangle / \mathrm{x} \in \mathrm{R}_{1}\right\}$ and $\mathrm{H}=\left\{\langle\mathrm{x}, \mu(\mathrm{x}), \eta(\mathrm{x})\rangle / \mathrm{x} \in \mathrm{R}_{2}\right\}$ be any two intuitionistic fuzzy sets defined on a semi rings $R_{1}$ and $R_{2}$ respectively.

Then, $\gamma^{\mathrm{G}}=\left\{\left\langle\mathrm{A}, \gamma^{\alpha}(\mathrm{A}), \gamma^{\beta}(\mathrm{A})\right\rangle / \mathrm{A} \in \mathfrak{R}_{1}\right\}$ and $\lambda^{\mathrm{H}}=\left\{\left\langle\mathrm{A}, \lambda^{\mu}(\mathrm{A}), \lambda^{\eta}(\mathrm{A})\right\rangle / \mathrm{A} \in \mathfrak{R}_{2}\right\}$ be any two intuitionistic fuzzy semi HX ring of a semi HX ring $\mathfrak{R}_{1}$ and $\mathfrak{R}_{2}$ respectively. Then,

$\left(\gamma^{\mathrm{G}} \times \lambda^{\mathrm{H}}\right)=\left\{\left\langle(\mathrm{A}, \mathrm{B}),\left(\gamma^{\alpha} \cap \lambda^{\mu}\right)(\mathrm{A}, \mathrm{B}),\left(\gamma^{\beta} \cup \lambda^{\eta}\right)(\mathrm{A}, \mathrm{B})\right\rangle /(\mathrm{A}, \mathrm{B}) \in \mathfrak{R}_{1} \times \mathfrak{R}_{2}\right\}$,

where $\quad\left(\gamma^{\alpha} \cap \lambda^{\mu}\right)(\mathrm{A}, \mathrm{B})=\min \left\{\gamma^{\alpha}(\mathrm{A}), \lambda^{\mu}(\mathrm{B})\right\}$, for all $(\mathrm{A}, \mathrm{B}) \in \mathfrak{R}_{1} \times \mathfrak{R}_{2}$,

$$
\left(\gamma^{\beta} \cup \lambda^{\eta}\right)(\mathrm{A}, \mathrm{B})=\max \left\{\gamma^{\beta}(\mathrm{A}), \lambda^{\eta}(\mathrm{B})\right\}, \text { for all }(\mathrm{A}, \mathrm{B}) \in \mathfrak{R}_{1} \times \mathfrak{R}_{2} .
$$

Let $\gamma^{\mathrm{G}} \times \lambda^{\mathrm{H}}$ be an intuitionistic fuzzy semi $\mathrm{HX}$ ring of $\mathfrak{R}_{1} \times \mathfrak{R}_{2}$ and $\mathrm{A}, \mathrm{B} \in \mathfrak{R}_{1}$ then $\left(\mathrm{A}, \mathrm{Q}^{1}\right),\left(\mathrm{B}, \mathrm{Q}^{1}\right) \in \mathfrak{R}_{1} \times \mathfrak{R}_{2}$.

Given,

$$
\lambda^{\mu}\left(\mathrm{Q}^{1}\right) \geq \gamma^{\alpha}(\mathrm{A}) \text { and } \lambda^{\eta}\left(\mathrm{Q}^{1}\right) \leq \gamma^{\beta}(\mathrm{A}) \text {, for all } \mathrm{A} \in \mathfrak{R}_{1} .
$$

i. $\gamma^{\alpha}(\mathrm{A}+\mathrm{B})=\min \left\{\gamma^{\alpha}(\mathrm{A}+\mathrm{B}), \lambda^{\mu}\left(\mathrm{Q}^{1}+\mathrm{Q}^{1}\right)\right\}$

$=\left(\gamma^{\alpha} \cap \lambda^{\mu}\right)\left(\mathrm{A}+\mathrm{B}, \mathrm{Q}^{1}+\mathrm{Q}^{1}\right)$

$=\quad\left(\gamma^{\alpha} \cap \lambda^{\mu}\right)\left(\left(\mathrm{A}, \mathrm{Q}^{1}\right)+\left(\mathrm{B}, \mathrm{Q}^{1}\right)\right)$

$\geq \quad \min \left\{\left(\gamma^{\alpha} \cap \lambda^{\mu}\right)\left(\mathrm{A}, \mathrm{Q}^{1}\right),\left(\gamma^{\alpha} \cap \lambda^{\mu}\right)\left(\mathrm{B}, \mathrm{Q}^{1}\right)\right\}$

$=\min \left\{\min \left\{\gamma^{\alpha}(\mathrm{A}), \lambda^{\mu}\left(\mathrm{Q}^{1}\right)\right\}, \min \left\{\gamma^{\alpha}(\mathrm{B}), \lambda^{\mu}\left(\mathrm{Q}^{1}\right)\right\}\right\}$

$=\min \left\{\gamma^{\alpha}(\mathrm{A}), \gamma^{\alpha}(\mathrm{B})\right\}$

ii.

$\gamma^{\alpha}(\mathrm{A}+\mathrm{B}) \quad \geq \quad \min \left\{\gamma^{\alpha}(\mathrm{A}), \gamma^{\alpha}(\mathrm{B})\right\}$.

ii. $\quad \gamma^{\alpha}(\mathrm{AB})=$

$\min \left\{\gamma^{\alpha}(\mathrm{AB}), \lambda^{\mu}\left(\mathrm{Q}^{1} \mathrm{Q}^{1}\right)\right\}$

$=\left(\gamma^{\alpha} \cap \lambda^{\mu}\right)\left(\mathrm{AB}, \mathrm{Q}^{1} \mathrm{Q}^{1}\right)$

$=\quad\left(\gamma^{\alpha} \cap \lambda^{\mu}\right)\left(\left(\mathrm{A}, \mathrm{Q}^{1}\right) \cdot\left(\mathrm{B}, \mathrm{Q}^{1}\right)\right)$

$\geq \quad \min \left\{\left(\gamma^{\alpha} \cap \lambda^{\mu}\right)\left(\mathrm{A}, \mathrm{Q}^{1}\right),\left(\gamma^{\alpha} \cap \lambda^{\mu}\right)\left(\mathrm{B}, \mathrm{Q}^{1}\right)\right\}$

$=\min \left\{\min \left\{\gamma^{\alpha}(\mathrm{A}), \lambda^{\mu}\left(\mathrm{Q}^{1}\right)\right\}, \min \left\{\gamma^{\alpha}(\mathrm{B}), \lambda^{\mu}\left(\mathrm{Q}^{1}\right)\right\}\right\}$

$=\min \left\{\gamma^{\alpha}(\mathrm{A}), \gamma^{\alpha}(\mathrm{B})\right\}$

$\gamma^{\alpha}(\mathrm{AB}) \geq \min \left\{\gamma^{\alpha}(\mathrm{A}), \gamma^{\alpha}(\mathrm{B})\right\}$.

iii. $\quad \gamma^{\beta}(\mathrm{A}+\mathrm{B})=\max \left\{\gamma^{\beta}(\mathrm{A}+\mathrm{B}), \lambda^{\eta}\left(\mathrm{Q}^{1}+\mathrm{Q}^{1}\right)\right\}$

$=\left(\gamma^{\beta} \cup \lambda^{\eta}\right)\left(A+B, Q^{1}+Q^{1}\right)$

$=\quad\left(\gamma^{\beta} \cup \lambda^{\eta}\right)\left(\left(\mathrm{A}, \mathrm{Q}^{1}\right)+\left(\mathrm{B}, \mathrm{Q}^{1}\right)\right)$

$\left.\leq \quad \max \left\{\left(\gamma^{\beta} \cup \lambda^{\eta}\right)\left(\mathrm{A}, \mathrm{Q}^{1}\right)\right),\left(\gamma^{\beta} \cup \lambda^{\eta}\right)\left(\mathrm{B}, \mathrm{Q}^{1}\right)\right\}$

$\leq \quad \max \left\{\max \left\{\gamma^{\beta}(\mathrm{A}), \lambda^{\eta}\left(\mathrm{Q}^{1}\right)\right\}, \max \left\{\gamma^{\beta}(\mathrm{B}), \lambda^{\eta}\left(\mathrm{Q}^{1}\right)\right\}\right\}$

$\leq \quad \max \left\{\gamma^{\beta}(\mathrm{A}), \gamma^{\beta}(\mathrm{B})\right\}$

$\gamma^{\beta}(\mathrm{A}+\mathrm{B}) \leq \max \left\{\gamma^{\beta}(\mathrm{A}), \gamma^{\beta}(\mathrm{B})\right\}$.

iv. $\quad \gamma^{\beta}(\mathrm{AB})=\max \left\{\gamma^{\beta}(\mathrm{AB}), \lambda^{\eta}\left(\mathrm{Q}^{1} \mathrm{Q}^{1}\right)\right\}$

$=\left(\gamma^{\beta} \cup \lambda^{\eta}\right)\left(\mathrm{AB}, \mathrm{Q}^{1} \mathrm{Q}^{1}\right)$

$=\quad\left(\gamma^{\beta} \cup \lambda^{\eta}\right)\left(\left(\mathrm{A}, \mathrm{Q}^{1}\right) \cdot\left(\mathrm{B}, \mathrm{Q}^{1}\right)\right)$

$\left.\leq \quad \max \left\{\left(\gamma^{\beta} \cup \lambda^{\eta}\right)\left(\mathrm{A}, \mathrm{Q}^{1}\right)\right),\left(\gamma^{\beta} \cup \lambda^{\eta}\right)\left(\mathrm{B}, \mathrm{Q}^{1}\right)\right\}$

$\leq \quad \max \left\{\max \left\{\gamma^{\beta}(\mathrm{A}), \lambda^{\eta}\left(\mathrm{Q}^{1}\right)\right\}, \max \left\{\gamma^{\beta}(\mathrm{B}), \lambda^{\eta}\left(\mathrm{Q}^{1}\right)\right\}\right\}$

$\leq \quad \max \left\{\gamma^{\beta}(\mathrm{A}), \gamma^{\beta}(\mathrm{B})\right\}$

Hence, $\gamma^{G}$ is an intuitionistic fuzzy semi HX ring of $\mathfrak{R}_{1}$.

$\max \left\{\gamma^{\beta}(\mathrm{A}), \gamma^{\beta}(\mathrm{B})\right\}$.

\subsection{Theorem}

Let $\mathrm{G}$ and $\mathrm{H}$ be any two intuitionistic fuzzy sets of $\mathrm{R}_{1}$ and $\mathrm{R}_{2}$ respectively. Let $\Re_{1} \subset 2^{\mathrm{R}}{ }_{1}-\{\phi\}$ and $\mathfrak{R}_{2} \subset 2^{\mathrm{R}}{ }_{2}-\{\phi\}$ be any two HX rings. If $\gamma^{\mathrm{G}}$ and $\lambda^{\mathrm{H}}$ are any two intuitionistic fuzzy semi HX rings 
of $\Re_{1}$ and $\Re_{2}$ respectively, such that $\gamma^{\alpha}(\mathrm{Q}) \geq \lambda^{\mu}(\mathrm{A})$ and $\gamma^{\beta}(\mathrm{Q}) \leq \lambda^{\eta}(\mathrm{A})$, for all $\mathrm{A} \in \mathfrak{R}_{2}$, Q being the identity element of $\mathfrak{R}_{1}$. If $\gamma^{\mathrm{G}} \times \lambda^{\mathrm{H}}$ is an intuitionistic fuzzy semi HX ring of $\Re_{1} \times \Re_{2}$, then $\lambda^{\mathrm{H}}$ is an intuitionistic fuzzy semi HX ring of $\Re_{2}$.

\section{Proof}

Let $\mathrm{G}=\left\{\langle\mathrm{x}, \alpha(\mathrm{x}), \beta(\mathrm{x})\rangle / \mathrm{x} \in \mathrm{R}_{1}\right\}$ and $\mathrm{H}=\left\{\langle\mathrm{x}, \mu(\mathrm{x}), \eta(\mathrm{x})\rangle / \mathrm{x} \in \mathrm{R}_{2}\right\}$ be any two intuitionistic fuzzy sets defined on a semi ring $R_{1}$ and $R_{2}$ respectively.

Then, $\gamma^{\mathrm{G}}=\left\{\left\langle\mathrm{A}, \gamma^{\alpha}(\mathrm{A}), \gamma^{\beta}(\mathrm{A})\right\rangle / \mathrm{A} \in \mathfrak{R}_{1}\right\}$ and $\lambda^{\mathrm{H}}=\left\{\left\langle\mathrm{A}, \lambda^{\mu}(\mathrm{A}), \lambda^{\eta}(\mathrm{A})\right\rangle / \mathrm{A} \in \mathfrak{R}_{2}\right\}$ be any two intuitionistic fuzzy semi HX ring of a semi HX ring $\mathfrak{R}_{1}$ and $\mathfrak{R}_{2}$ respectively. Then,

$\left(\gamma^{\mathrm{G}} \times \lambda^{\mathrm{H}}\right)=\left\{\left\langle(\mathrm{A}, \mathrm{B}),\left(\gamma^{\alpha} \cap \lambda^{\mu}\right)(\mathrm{A}, \mathrm{B}),\left(\gamma^{\beta} \cup \lambda^{\eta}\right)(\mathrm{A}, \mathrm{B})\right\rangle /(\mathrm{A}, \mathrm{B}) \in \mathfrak{R}_{1} \times \mathfrak{R}_{2}\right\}$,

where $\quad\left(\gamma^{\alpha} \cap \lambda^{\mu}\right)(\mathrm{A}, \mathrm{B})=\quad \min \left\{\gamma^{\alpha}(\mathrm{A}), \lambda^{\mu}(\mathrm{B})\right\}$, for all $(\mathrm{A}, \mathrm{B}) \in \mathfrak{R}_{1} \times \mathfrak{R}_{2}$,

$$
\left(\gamma^{\beta} \cup \lambda^{\eta}\right)(\mathrm{A}, \mathrm{B})=\max \left\{\gamma^{\beta}(\mathrm{A}), \lambda^{\eta}(\mathrm{B})\right\} \text {, for all }(\mathrm{A}, \mathrm{B}) \in \mathfrak{R}_{1} \times \mathfrak{R}_{2} \text {. }
$$

Let $\gamma^{\mathrm{G}} \times \lambda^{\mathrm{H}}$ be an intuitionistic fuzzy semi $\mathrm{HX}$ ring of $\mathfrak{R}_{1} \times \mathfrak{R}_{2}$ and $\mathrm{A}, \mathrm{B} \in \mathfrak{R}_{2}$ then $(\mathrm{Q}, \mathrm{A}),(\mathrm{Q}, \mathrm{B}) \in \mathfrak{R}_{1} \times \mathfrak{R}_{2}$.

Given, $\gamma^{\alpha}(\mathrm{Q}) \geq \lambda^{\mu}(\mathrm{A})$ and $\gamma^{\beta}(\mathrm{Q})$

i. $\quad \lambda^{\mu}(\mathrm{A}+\mathrm{B})$

$\leq \lambda^{\eta}(\mathrm{A})$, for all $\mathrm{A} \in \mathfrak{R}_{2}$

$=\min \left\{\gamma^{\alpha}(\mathrm{Q}+\mathrm{Q}), \lambda^{\mu}(\mathrm{A}+\mathrm{B})\right\}$

$=\quad\left(\gamma^{\alpha} \cap \lambda^{\mu}\right)(\mathrm{Q}+\mathrm{Q}, \mathrm{A}+\mathrm{B})$

$=\quad\left(\gamma^{\alpha} \cap \lambda^{\mu}\right)((\mathrm{Q}, \mathrm{A})+(\mathrm{Q}, \mathrm{B}))$

$\geq \quad \min \left\{\left(\gamma^{\alpha} \cap \lambda^{\mu}\right)(\mathrm{Q}, \mathrm{A}),\left(\gamma^{\alpha} \cap \lambda^{\mu}\right)(\mathrm{Q}, \mathrm{B})\right\}$

$=\min \left\{\min \left\{\gamma^{\alpha}(\mathrm{Q}), \lambda^{\mu}(\mathrm{A})\right\}, \min \left\{\gamma^{\alpha}(\mathrm{Q}), \lambda^{\mu}(\mathrm{A})\right\}\right\}$

$\begin{array}{lll}\lambda^{\mu}(\mathrm{A}+\mathrm{B}) & =\min \left\{\lambda^{\mu}(\mathrm{A}), \lambda^{\mu}(\mathrm{B})\right\} \\ & \geq & \min \left\{\lambda^{\mu}(\mathrm{A}), \lambda^{\mu}(\mathrm{B})\right\}\end{array}$

ii. $\quad \lambda^{\mu}(\mathrm{AB}) \quad \min \left\{\gamma^{\alpha}(\mathrm{QQ}), \lambda^{\mu}(\mathrm{AB})\right\}$

$=\left(\gamma^{\alpha} \cap \lambda^{\mu}\right)(\mathrm{QQ}, \mathrm{AB})$

$=\left(\gamma^{\alpha} \cap \lambda^{\mu}\right)((\mathrm{Q}, \mathrm{A}) \cdot(\mathrm{Q}, \mathrm{A}))$

$\geq \quad \min \left\{\left(\gamma^{\alpha} \cap \lambda^{\mu}\right)(\mathrm{Q}, \mathrm{A}),\left(\gamma^{\alpha} \cap \lambda^{\mu}\right)(\mathrm{Q}, \mathrm{A})\right\}$

$=\min \left\{\min \left\{\gamma^{\alpha}(\mathrm{Q}), \lambda^{\mu}(\mathrm{A})\right\}, \min \left\{\gamma^{\alpha}(\mathrm{Q}), \lambda^{\mu}(\mathrm{B})\right\}\right\}$

$=\min \left\{\lambda^{\mu}(\mathrm{A}), \lambda^{\mu}(\mathrm{B})\right\}$

$\lambda^{\mu}(\mathrm{AB}) \geq \min \left\{\lambda^{\mu}(\mathrm{A}), \lambda^{\mu}(\mathrm{B})\right\}$

iii. $\quad \lambda^{\eta}(\mathrm{A}+\mathrm{B}) \quad=\max \left\{\gamma^{\beta}(\mathrm{Q}+\mathrm{Q}), \lambda^{\eta}(\mathrm{A}+\mathrm{B})\right\}$

$=\left(\gamma^{\beta} \cup \lambda^{\eta}\right)(\mathrm{Q}+\mathrm{Q}, \mathrm{A}+\mathrm{B})$

$=\quad\left(\gamma^{\beta} \cup \lambda^{\eta}\right)((\mathrm{Q}, \mathrm{A})-(\mathrm{Q}, \mathrm{B}))$

$\leq \quad \max \left\{\left(\gamma^{\beta} \cup \lambda^{\eta}\right)(\mathrm{Q}, \mathrm{A}),\left(\gamma^{\beta} \cup \lambda^{\eta}\right)(\mathrm{Q}, \mathrm{B})\right\}$

$\leq \quad \max \left\{\max \left\{\gamma^{\beta}(\mathrm{Q}), \lambda^{\eta}(\mathrm{A})\right\}, \max \left\{\gamma^{\beta}(\mathrm{Q}), \lambda^{\eta}(\mathrm{B})\right\}\right\}$

$\leq \quad \max \left\{\lambda^{\eta}(\mathrm{A}), \lambda^{\eta}(\mathrm{B})\right\}$

$\begin{array}{llll} & \lambda^{\eta}(\mathrm{A}+\mathrm{B}) & \leq & \max \left\{\lambda^{\eta}(\mathrm{A}), \lambda^{\eta}(\mathrm{B})\right\} . \\ \text { iv. } \quad \lambda^{\eta}(\mathrm{AB}) & = & \max \left\{\gamma^{\beta}(\mathrm{QQ}), \lambda^{\eta}(\mathrm{AB})\right\}\end{array}$

$\begin{array}{ll}= & \max \left\{\gamma^{\beta}(\mathrm{QQ}), \lambda^{\eta}(\mathrm{AB})\right. \\ = & \left(\gamma^{\beta} \cup \lambda^{\eta}\right)(\mathrm{QQ}, \mathrm{AB})\end{array}$

$=\quad\left(\gamma^{\beta} \cup \lambda^{\eta}\right)((\mathrm{Q}, \mathrm{A}) \cdot(\mathrm{Q}, \mathrm{B}))$

$\leq \quad \max \left\{\left(\gamma^{\beta} \cup \lambda^{\eta}\right)(\mathrm{Q}, \mathrm{A}),\left(\gamma^{\beta} \cup \lambda^{\eta}\right)(\mathrm{Q}, \mathrm{B})\right\}$

$\leq \quad \max \left\{\max \left\{\gamma^{\beta}(\mathrm{Q}), \lambda^{\eta}(\mathrm{A})\right\}, \max \left\{\gamma^{\beta}(\mathrm{Q}), \lambda^{\eta}(\mathrm{B})\right\}\right\}$

$\leq \quad \max \left\{\lambda^{\eta}(\mathrm{A}), \lambda^{\eta}(\mathrm{B})\right\}$

$\lambda^{\eta}(\mathrm{AB}) \leq \max \left\{\lambda^{\eta}(\mathrm{A}), \lambda^{\eta}(\mathrm{B})\right\}$.

Hence, $\lambda^{\mathrm{H}}$ is an intuitionistic fuzzy semi HX ring of $\mathfrak{R}_{2}$.

\subsection{Corollary}

Let $\mathrm{G}$ and $\mathrm{H}$ be any two intuitionistic fuzzy sets of $\mathrm{R}_{1}$ and $\mathrm{R}_{2}$ respectively. Let $\Re_{1} \subset 2^{\mathrm{R}}{ }_{1}-\{\phi\}$ and $\Re_{2} \subset 2^{\mathrm{R}}{ }_{2}-\{\phi\}$ be any two semi HX rings. If $\gamma^{\mathrm{G}}$ and $\lambda^{\mathrm{H}}$ are any two intuitionistic fuzzy semi HX rings of $\Re_{1}$ and $\Re_{2}$ respectively. If $\gamma^{\mathrm{G}} \times \lambda^{\mathrm{H}}$ is an intuitionistic fuzzy semi HX ring of $\mathfrak{R}_{1} \times \Re_{2}$, then either $\gamma^{\mathrm{G}}$ is an intuitionistic fuzzy semi HX ring of $\mathfrak{R}_{1}$ or $\lambda^{\mathrm{H}}$ is an intuitionistic fuzzy semi HX ring of $\mathfrak{R}_{2}$. 


\subsection{Definition}

Let $\mathrm{H}$ be an intuitionistic fuzzy set of R. Let $\Re \subset 2^{\mathrm{R}}-\{\phi\}$ be a semi HX ring. Let $\lambda^{\mathrm{H}}$ be an intuitionistic fuzzy set of $\mathfrak{R}$. We define the following "necessity" and possibility" operations:

$$
\begin{aligned}
& \square \lambda^{\mathrm{H}}=\left\{\left\langle\mathrm{A}, \lambda^{\mu}(\mathrm{A}), 1-\lambda^{\mu}(\mathrm{A})\right\rangle / \mathrm{A} \in \mathfrak{R}\right\} \\
& \Delta \lambda^{\mathrm{H}} \quad=\left\{\left\langle\mathrm{A}, 1-\lambda^{\eta}(\mathrm{A}), \lambda^{\eta}(\mathrm{A})\right\rangle / \mathrm{A} \in \mathfrak{R}\right\} .
\end{aligned}
$$

\subsection{Theorem}

Let $\mathrm{H}$ be an intuitionistic fuzzy set on R. Let $\lambda^{\mathrm{H}}$ be an intuitionistic fuzzy semi HX ring of a semi HX ring $\Re$ then $\square \lambda^{\mathrm{H}}$ is an intuitionistic fuzzy semi HX ring of a semi HX ring $\mathfrak{R}$.

\section{Proof}

Let $\lambda^{\mathrm{H}}$ be an intuitionistic fuzzy semi HX ring of a semi HX ring $\Re$. Then,

$\begin{array}{lllll}\text { i. } & \lambda^{\mu}(\mathrm{A}+\mathrm{B}) & \geq & \min \left\{\lambda^{\mu}(\mathrm{A}), \lambda^{\mu}(\mathrm{B})\right\}, \\ \text { ii. } & \lambda^{\mu}(\mathrm{AB}) & \geq & \min \left\{\lambda^{\mu}(\mathrm{A}), \lambda^{\mu}(\mathrm{B})\right\}, \\ \text { iii. } & \lambda^{\eta}(\mathrm{A}+\mathrm{B}) & \leq & \max \left\{\lambda^{\eta}(\mathrm{A}), \lambda^{\eta}(\mathrm{B})\right\}, \\ \text { iv. } \quad \lambda^{\eta}(\mathrm{AB}) & \leq & \max \left\{\lambda^{\eta}(\mathrm{A}), \lambda^{\eta}(\mathrm{B})\right\} . \\ \text { Now, } & & \lambda^{\mu}(\mathrm{A}+\mathrm{B}) & \geq & \min \left\{\lambda^{\mu}(\mathrm{A}), \lambda^{\mu}(\mathrm{B})\right\} \\ & 1-\lambda^{\mu}(\mathrm{A}+\mathrm{B}) & \leq & 1-\min \left\{\lambda^{\mu}(\mathrm{A}), \lambda^{\mu}(\mathrm{B})\right\} \\ & & \leq & \max \left\{1-\lambda^{\mu}(\mathrm{A}), 1-\lambda^{\mu}(\mathrm{B})\right\} . \\ \text { That is, } & 1-\lambda^{\mu}(\mathrm{A}+\mathrm{B}) \leq & \max \left\{1-\lambda^{\mu}(\mathrm{A}), 1-\lambda^{\mu}(\mathrm{B})\right\} . \\ \text { We have, } & \lambda^{\mu}(\mathrm{AB}) & \geq & \min \left\{\lambda^{\mu}(\mathrm{A}), \lambda^{\mu}(\mathrm{B})\right\} \\ & 1-\lambda^{\mu}(\mathrm{AB}) & \leq & 1-\min \left\{\lambda^{\mu}(\mathrm{A}), \lambda^{\mu}(\mathrm{B})\right\} \\ & & & \leq & \max \left\{1-\lambda^{\mu}(\mathrm{A}), 1-\lambda^{\mu}(\mathrm{B})\right\} . \\ \text { That is, } & 1-\lambda^{\mu}(\mathrm{AB}) & \leq & \max \left\{1-\lambda^{\mu}(\mathrm{A}), 1-\lambda^{\mu}(\mathrm{B})\right\} .\end{array}$

\subsection{Theorem}

Hence, $\square \lambda^{\mathrm{H}}$ is an intuitionistic fuzzy semi HX ring of a semi HX ring $\mathfrak{R}$.

Let $\mathrm{H}$ be an intuitionistic fuzzy set on R. Let $\lambda^{\mathrm{H}}$ be an intuitionistic fuzzy semi $\mathrm{HX}$ ring of a semi HX ring $\Re$ then $\Delta \lambda^{\mathrm{H}}$ is an intuitionistic fuzzy semi HX ring of a semi HX ring $\mathfrak{R}$.

Proof

Let $\lambda^{\mathrm{H}}$ be an intuitionistic fuzzy semi HX ring of a semi HX ring $\Re$. Then,

$$
\begin{array}{llll}
\text { i. } \quad & \lambda^{\mu}(\mathrm{A}+\mathrm{B}) & \geq & \min \left\{\lambda^{\mu}(\mathrm{A}), \lambda^{\mu}(\mathrm{B})\right\}, \\
\text { ii. } \quad \lambda^{\mu}(\mathrm{AB}) & \geq & \min \left\{\lambda^{\mu}(\mathrm{A}), \lambda^{\mu}(\mathrm{B})\right\}, \\
\text { iii. } \quad \lambda^{\eta}(\mathrm{A}+\mathrm{B}) & \leq & \max \left\{\lambda^{\eta}(\mathrm{A}), \lambda^{\eta}(\mathrm{B})\right\}, \\
\text { iv. } \quad \lambda^{\eta}(\mathrm{AB}) & \leq & \max \left\{\lambda^{\eta}(\mathrm{A}), \lambda^{\eta}(\mathrm{B})\right\} . \\
\text { Now, } \quad \lambda^{\eta}(\mathrm{A}+\mathrm{B}) & \leq & \max \left\{\lambda^{\eta}(\mathrm{A}), \lambda^{\eta}(\mathrm{B})\right\} \\
& 1-\lambda^{\eta}(\mathrm{A}+\mathrm{B}) & \geq & 1-\max \left\{\lambda^{\eta}(\mathrm{A}), \lambda^{\eta}(\mathrm{B})\right\} \\
& \geq & \min \left\{1-\lambda^{\eta}(\mathrm{A}), 1-\lambda^{\eta}(\mathrm{B})\right\} . \\
\text { That is, } 1-\lambda^{\eta}(\mathrm{A}+\mathrm{B}) & \geq & \min \left\{1-\lambda^{\eta}(\mathrm{A}), 1-\lambda^{\eta}(\mathrm{B})\right\} . \\
\text { We have, } & & \\
\quad \lambda^{\eta}(\mathrm{AB}) & \leq & \max \left\{\lambda^{\eta}(\mathrm{A}), \lambda^{\eta}(\mathrm{B})\right\} \\
\quad 1-\lambda^{\eta}(\mathrm{AB}) & \geq & 1-\max \left\{\lambda^{\eta}(\mathrm{A}), \lambda^{\eta}(\mathrm{B})\right\} \\
& & \min \left\{1-\lambda^{\eta}(\mathrm{A}), 1-\lambda^{\eta}(\mathrm{B})\right\} .
\end{array}
$$

That is, $1-\lambda^{\eta}(\mathrm{AB}) \quad \geq \min \left\{1-\lambda^{\eta}(\mathrm{A}), 1-\lambda^{\eta}(\mathrm{B})\right\}$.

Hence, $\Delta \lambda^{\mathrm{H}}$ is an intuitionistic fuzzy HX subring of a HX ring $\Re$.

\section{HOMOMORPHISM AND ANTI HOMOMORPHISM OF AN INTUITIONISTIC FUZZY SEMI HX RING OF A SEMI HX RING}

In this section, we introduce the concept of an image, pre-image of an intuitionistic fuzzy subset of a semi HX ring and discuss the properties of homomorphic and anti homomorphic images and pre images of an intuitionistic fuzzy semi HX ring of a semi HX ring $\mathfrak{R}$. 


\subsection{Definition}

Let $R_{1}$ and $R_{2}$ be any two semi rings. Let $\Re_{1} \subset 2{ }^{R}{ }_{1}-\{\phi\}$ and $\Re_{2} \subset 2{ }^{R}{ }_{2}-\{\phi\}$ be any two semi $\mathrm{HX}$ rings defined on $\mathrm{R}_{1}$ and $\mathrm{R}_{2}$ respectively. Let $\mathrm{H}$ and $\mathrm{G}$ be any two intuitionistic fuzzy subsets in $R_{1}$ and $R_{2}$ respectively. Let $\lambda^{\mathrm{H}}$ and $\gamma^{\mathrm{G}}$ be fuzzy subsets defined on $\mathfrak{R}_{1}$ and $\mathfrak{R}_{2}$ respectively induced by $\mathrm{H}$ and $\mathrm{G}$. Let $\mathrm{f}: \Re_{1} \rightarrow \Re_{2}$ be a mapping then the image of $\lambda^{\mathrm{H}}$ denoted as $\mathrm{f}\left(\lambda^{\mathrm{H}}\right)$ is an intuitionistic fuzzy subset of $\mathfrak{R}_{2}$ defined as for each $\mathrm{U} \in \mathfrak{R}_{2}$,

$$
\begin{array}{ll}
\left(\mathrm{f}\left(\lambda^{\mu}\right)\right)(\mathrm{U})= & \begin{cases}\sup \left\{\lambda^{\mu}(\mathrm{X}): \mathrm{X} \in \mathrm{f}^{-1}(\mathrm{U})\right\} & \text { if } \mathrm{f}^{-1}(\mathrm{U}) \neq \phi \\
0 & \text { otherwise }\end{cases} \\
\left(\mathrm{f}\left(\lambda^{\eta}\right)\right)(\mathrm{U})= & \begin{cases}\inf \left\{\lambda^{\eta}(\mathrm{X}): \mathrm{X} \in \mathrm{f}^{-1}(\mathrm{U})\right\} & \text { if } \mathrm{f}^{-1}(\mathrm{U}) \neq \phi \\
1 & \text { otherwise }\end{cases}
\end{array}
$$

Also the pre-image of $\gamma^{\mathrm{G}}$ denoted as $\mathrm{f}^{-1}\left(\gamma^{\mathrm{G}}\right)$ under $\mathrm{f}$ is an intuitionistic fuzzy subset of $\mathfrak{R}_{1}$ defined as for each $X \in \mathfrak{R}_{1},\left(f^{-1}\left(\gamma^{\alpha}\right)\right)(X)=\gamma^{\alpha}(f(X))$ and $\left(f^{-1}\left(\gamma^{\beta}\right)\right)(X)=\gamma^{\beta}(f(X))$.

\subsection{Definition}

Let $R_{1}$ and $R_{2}$ be any two semi rings. Let $\Re_{1} \subset 2{ }^{R}{ }_{1}-\{\phi\}$ and $\Re_{2} \subset 2{ }^{R}{ }_{2}-\{\phi\}$ be any two semi $H X$ rings defined on $R_{1}$ and $R_{2}$ respectively. The mapping f: $\Re_{1} \rightarrow \Re_{2}$ is said to be a homomorphism if it satisfies the following conditions. For any $\mathrm{A}, \mathrm{B} \in \mathfrak{R}_{1}$,

$$
\begin{array}{llll}
\text { i. } & \mathrm{f}(\mathrm{A}+\mathrm{B}) & \mathrm{f}(\mathrm{A})+\mathrm{f}(\mathrm{B}) \\
\text { ii. } \mathrm{f}(\mathrm{AB}) & = & \mathrm{f}(\mathrm{A}) \cdot \mathrm{f}(\mathrm{B}) .
\end{array}
$$

\subsection{Definition}

Let $R_{1}$ and $R_{2}$ be any two rings. Let $\mathfrak{R}_{1} \subset 2^{\mathrm{R} 1}-\{\phi\}$ and $\mathfrak{R}_{2} \subset 2^{\mathrm{R} 2}-\{\phi\}$ be any two semi HX rings defined on $R_{1}$ and $R_{2}$ respectively(not necessarily commutative). The mapping $f: \Re_{1} \rightarrow \mathfrak{R}_{2}$ is said to be an anti homomorphism if it satisfies the following conditions. For any $\mathrm{A}, \mathrm{B} \in \mathfrak{R}_{1}$,

$$
\begin{array}{llll}
\text { i. } & \mathrm{f}(\mathrm{A}+\mathrm{B}) & \mathrm{f}(\mathrm{A})+\mathrm{f}(\mathrm{B}) \\
\text { ii. } \mathrm{f}(\mathrm{AB}) & = & \mathrm{f}(\mathrm{B}) \cdot \mathrm{f}(\mathrm{A}) .
\end{array}
$$

\subsection{Theorem}

Let $\Re_{1}$ and $\Re_{2}$ be any two semi $\mathrm{HX}$ rings on the rings $\mathrm{R}_{1}$ and $\mathrm{R}_{2}$ respectively. Let $\mathrm{f}: \mathfrak{R}_{1} \rightarrow \mathfrak{R}_{2}$ be a homomorphism onto semi HX rings. Let $\mathrm{H}$ be an intuitionistic fuzzy subset of $\mathrm{R}_{1}$. Let $\lambda^{\mathrm{H}}$ be an intuitionistic fuzzy semi HX ring of $\mathfrak{R}_{1}$ then $\mathrm{f}\left(\lambda^{\mathrm{H}}\right)$ is an intuitionistic fuzzy semi HX ring of $\Re_{2}$, if $\lambda^{\mathrm{H}}$ has a supremum property and $\lambda^{\mathrm{H}}$ is f-invariant.

\section{Proof}

Let $\mathrm{H}=\left\{\langle\mathrm{x}, \mu(\mathrm{x}), \eta(\mathrm{x})\rangle / \mathrm{x} \in \mathrm{R}_{1}\right\}$ be an intuitionistic fuzzy sets defined on a semi ring $\mathrm{R}_{1}$. Then, $\lambda^{\mathrm{H}}=\left\{\left\langle\mathrm{X}, \lambda^{\mu}(\mathrm{X}), \lambda^{\eta}(\mathrm{X})\right\rangle / \mathrm{X} \in \mathfrak{R}_{1}\right\}$ be an intuitionistic fuzzy semi HX ring of a semi HX ring $\Re_{1}$. Then, $\mathrm{f}\left(\lambda^{\mathrm{H}}\right)=\left\{\left\langle\mathrm{f}(\mathrm{X}), \mathrm{f}\left(\lambda^{\mu}\right)(\mathrm{f}(\mathrm{X})), \mathrm{f}\left(\lambda^{\eta}\right)(\mathrm{f}(\mathrm{X}))\right\rangle / \mathrm{X} \in \mathfrak{R}_{1}\right\}$.

There exist $\mathrm{X}, \mathrm{Y} \in \mathfrak{R}_{1}$ such that $\mathrm{f}(\mathrm{X}), \mathrm{f}(\mathrm{Y}) \in \mathfrak{R}_{2}$,

$\begin{array}{rlll}\text { i. } \quad\left(\mathrm{f}\left(\lambda^{\mu}\right)\right)(\mathrm{f}(\mathrm{X})+\mathrm{f}(\mathrm{Y})) & = & \left(\mathrm{f}\left(\lambda^{\mu}\right)\right)(\mathrm{f}(\mathrm{X}+\mathrm{Y})), \\ & = & \lambda^{\mu}(\mathrm{X}+\mathrm{Y}) \\ & \geq & \min \left\{\lambda^{\mu}(\mathrm{X}), \lambda^{\mu}(\mathrm{Y})\right\} \\ & = & \min \left\{\left(\mathrm{f}\left(\lambda^{\mu}\right)\right)(\mathrm{f}(\mathrm{X})),\left(\mathrm{f}\left(\lambda^{\mu}\right)\right)(\mathrm{f}(\mathrm{Y}))\right\} \\ & \left(\mathrm{f}\left(\lambda^{\mu}\right)\right)(\mathrm{f}(\mathrm{X})+\mathrm{f}(\mathrm{Y})) & \geq & \min \left\{\left(\mathrm{f}\left(\lambda^{\mu}\right)\right)(\mathrm{f}(\mathrm{X})),\left(\mathrm{f}\left(\lambda^{\mu}\right)\right)(\mathrm{f}(\mathrm{Y}))\right\} . \\ \left.\text { ii. } \quad\left(\mathrm{f}\left(\lambda^{\mu}\right)\right)(\mathrm{f}(\mathrm{X}) \mathrm{f}(\mathrm{Y}))\right) & = & \left(\mathrm{f}\left(\lambda^{\mu}\right)\right)(\mathrm{f}(\mathrm{XY})) \\ & = & \lambda^{\mu}(\mathrm{XY}) \\ & \geq & \min \left\{\lambda^{\mu}(\mathrm{X}), \lambda^{\mu}(\mathrm{Y})\right\} \\ & = & \min \left\{\left(\mathrm{f}\left(\lambda^{\mu}\right)\right)(\mathrm{f}(\mathrm{X})),\left(\mathrm{f}\left(\lambda^{\mu}\right)\right)(\mathrm{f}(\mathrm{Y}))\right\}\end{array}$




$\begin{array}{rlll}\left(\mathrm{f}\left(\lambda^{\mu}\right)\right)(\mathrm{f}(\mathrm{X}) \mathrm{f}(\mathrm{Y})) & \geq & \min \left\{\left(\mathrm{f}\left(\lambda^{\mu}\right)\right)(\mathrm{f}(\mathrm{X})),\left(\mathrm{f}\left(\lambda^{\mu}\right)\right)(\mathrm{f}(\mathrm{Y}))\right\} \\ \text { iii. } \quad\left(\mathrm{f}\left(\lambda^{\eta}\right)\right)(\mathrm{f}(\mathrm{X})+\mathrm{f}(\mathrm{Y})) & = & \left(\mathrm{f}\left(\lambda^{\eta}\right)\right)(\mathrm{f}(\mathrm{X}+\mathrm{Y})), \\ & = & \lambda^{\eta}(\mathrm{X}+\mathrm{Y}) \\ & \leq & \max \left\{\lambda^{\eta}(\mathrm{X}), \lambda^{\eta}(\mathrm{Y})\right\} \\ & = & \max \left\{\left(\mathrm{f}\left(\lambda^{\eta}\right)\right)(\mathrm{f}(\mathrm{X})),\left(\mathrm{f}\left(\lambda^{\eta}\right)\right)(\mathrm{f}(\mathrm{Y}))\right\} \\ & \left(\mathrm{f}\left(\lambda^{\eta}\right)\right)(\mathrm{f}(\mathrm{X})+\mathrm{f}(\mathrm{Y})) & \leq & \max \left\{\left(\mathrm{f}\left(\lambda^{\eta}\right)\right)(\mathrm{f}(\mathrm{X})),\left(\mathrm{f}\left(\lambda^{\eta}\right)\right)(\mathrm{f}(\mathrm{Y}))\right\} \\ \text { iv. } \quad\left(\mathrm{f}\left(\lambda^{\eta}\right)\right)(\mathrm{f}(\mathrm{X}) \mathrm{f}(\mathrm{Y})) & = & \left(\mathrm{f}\left(\lambda^{\eta}\right)\right)(\mathrm{f}(\mathrm{XY})) \\ & & & \lambda^{\eta}(\mathrm{XY}) \\ & & & \max \left\{\lambda^{\eta}(\mathrm{X}), \lambda^{\eta}(\mathrm{Y})\right\} \\ & = & \max \left\{\left(\mathrm{f}\left(\lambda^{\eta}\right)\right)(\mathrm{f}(\mathrm{X})),\left(\mathrm{f}\left(\lambda^{\eta}\right)\right)(\mathrm{f}(\mathrm{Y}))\right\} \\ & & & \\ & & & \\ & & & \end{array}$

Hence, $f\left(\lambda^{\mathrm{H}}\right)$ is an intuitionistic fuzzy semi HX ring of $\Re_{2}$.

\subsection{Theorem}

Let $\Re_{1}$ and $\Re_{2}$ be any two semi HX rings on $R_{1}$ and $R_{2}$ respectively. Let $f: \Re_{1} \rightarrow \Re_{2}$ be a homomorphism on semi HX rings. Let $G$ be an intuitionistic fuzzy subset of $R_{2}$. Let $\gamma^{G}$ be an intuitionistic fuzzy semi HX ring of $\mathfrak{R}_{2}$, then $\mathrm{f}^{-1}\left(\gamma^{\mathrm{G}}\right)$ is an intuitionistic fuzzy semi HX ring of $\mathfrak{R}_{1}$.

\section{Proof}

Let $\mathrm{G}=\left\{\langle\mathrm{y}, \alpha(\mathrm{y}), \beta(\mathrm{y})\rangle / \mathrm{y} \in \mathrm{R}_{2}\right\}$ be an intuitionistic fuzzy sets defined on a semi ring $\mathrm{R}_{2}$. Then, $\gamma^{\mathrm{G}}=\left\{\left\langle\mathrm{Y}, \gamma^{\alpha}(\mathrm{Y}), \gamma^{\beta}(\mathrm{Y})\right\rangle / \mathrm{Y} \in \mathfrak{R}_{2}\right\}$ be an intuitionistic fuzzy semi HX ring of a semi HX ring $\mathfrak{R}_{2}$. Then, $\mathrm{f}^{-1}\left(\gamma^{\mathrm{G}}\right)=\left\{\left\langle\mathrm{X}, \mathrm{f}^{-1}\left(\gamma^{\alpha}\right)(\mathrm{X}), \mathrm{f}^{-1}\left(\gamma^{\beta}\right)(\mathrm{X})\right\rangle / \mathrm{X} \in \mathfrak{R}_{1}\right\}$.

For any $\mathrm{X}, \mathrm{Y} \in \mathfrak{R}_{1}, \mathrm{f}(\mathrm{X}), \mathrm{f}(\mathrm{Y}) \in \mathfrak{R}_{2}$,

i.

$$
\begin{array}{rll}
\left(\mathrm{f}^{-1}\left(\gamma^{\alpha}\right)\right)(\mathrm{X}+\mathrm{Y}) & = & \gamma^{\alpha}(\mathrm{f}(\mathrm{X}+\mathrm{Y})) \\
& = & \gamma^{\alpha}(\mathrm{f}(\mathrm{X})+\mathrm{f}(\mathrm{Y}))
\end{array}
$$$$
\geq \quad \min \left\{\gamma^{\alpha}(\mathrm{f}(\mathrm{X})), \gamma^{\alpha}(\mathrm{f}(\mathrm{Y}))\right\}
$$

$\begin{array}{rlrl} & = & \min \left\{\left(\mathrm{f}^{-1}\left(\gamma^{\alpha}\right)\right)(\mathrm{X}),\left(\mathrm{f}^{-1}\left(\gamma^{\alpha}\right)\right)(\mathrm{Y})\right\} \\ \left(\mathrm{f}^{-1}\left(\gamma^{\alpha}\right)\right)(\mathrm{X}+\mathrm{Y}) \geq & \min \left\{\left(\mathrm{f}^{-1}\left(\gamma^{\alpha}\right)\right)(\mathrm{X}),\left(\mathrm{f}^{-1}\left(\gamma^{\alpha}\right)\right)(\mathrm{Y})\right\} .\end{array}$

$\begin{array}{rlrl}\left(\mathrm{f}^{-1}\left(\gamma^{\alpha}\right)\right)(\mathrm{X}+\mathrm{Y}) & \geq & \min \left\{\left(\mathrm{f}^{-1}\left(\gamma^{\alpha}\right)\right)\right. \\ \text { ii. } \quad & \left(\mathrm{f}^{-1}\left(\gamma^{\alpha}\right)\right)(\mathrm{XY}) & = & \gamma^{\alpha}(\mathrm{f}(\mathrm{XY}))\end{array}$

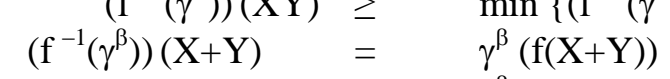$$
=\quad \gamma^{\alpha}(\mathrm{f}(\mathrm{X}) \mathrm{f}(\mathrm{Y}))
$$$$
\geq \quad \min \left\{\gamma^{\alpha}(\mathrm{f}(\mathrm{X})), \gamma^{\alpha}(\mathrm{f}(\mathrm{Y}))\right\}
$$

$\left(\mathrm{f}^{-1}\left(\gamma^{\alpha}\right)\right)(\mathrm{XY}) \geq \min \left\{\left(\mathrm{f}^{-1}\left(\gamma^{\alpha}\right)\right)(\mathrm{X}),\left(\mathrm{f}^{-1}\left(\gamma^{\alpha}\right)\right)(\mathrm{Y})\right\}$

$\begin{aligned}\left(\mathrm{f}^{-1}\left(\gamma^{\beta}\right)\right)(\mathrm{X}+\mathrm{Y}) & = & \gamma^{\beta}(\mathrm{f}(\mathrm{X}+\mathrm{Y})) \\ & = & \left.\gamma^{\beta}(\mathrm{X})+\mathrm{f}(\mathrm{Y})\right)\end{aligned}$

$\leq \quad \max \left\{\gamma^{\beta}(\mathrm{f}(\mathrm{X})), \gamma^{\beta}(\mathrm{f}(\mathrm{Y}))\right\}$

$\begin{array}{rlrl}\left(\mathrm{f}^{-1}\left(\gamma^{\beta}\right)\right)(\mathrm{X}+\mathrm{Y}) & \leq & \max \left\{\left(\mathrm{f}^{-1}\left(\eta^{\beta}\right)\right)(\mathrm{X}),\left(\mathrm{f}^{-1}\left(\gamma^{\beta}\right)\right)(\mathrm{Y})\right\} . \\ \text { iv. } \quad & \left(\mathrm{f}^{-1}\left(\gamma^{\beta}\right)\right)(\mathrm{XY}) & = & \gamma^{\beta}(\mathrm{f}(\mathrm{XY}))\end{array}$

$\begin{aligned}= & \gamma^{\beta}(\mathrm{f}(\mathrm{X}) \mathrm{f}(\mathrm{Y}))\end{aligned}$

$\leq \quad \max \left\{\gamma^{\beta}(\mathrm{f}(\mathrm{X})), \gamma^{\beta}(\mathrm{f}(\mathrm{Y}))\right\}$

$\begin{array}{lll}\left(\mathrm{f}^{-1}\left(\gamma^{\beta}\right)\right)(\mathrm{XY}) & =\max \left\{\left(\mathrm{f}^{-1}\left(\gamma^{\beta}\right)\right)(\mathrm{X}),\left(\mathrm{f}^{-1}\left(\gamma^{\beta}\right)\right)(\mathrm{Y})\right\} \\ & \leq & \max \left\{\left(\mathrm{f}^{-1}\left(\eta^{\beta}\right)\right)(\mathrm{X}),\left(\mathrm{f}^{-1}\left(\gamma^{\beta}\right)\right)(\mathrm{Y})\right\} .\end{array}$

Hence, $f^{-1}\left(\gamma^{G}\right)$ is an intuitionistic fuzzy semi HX ring of $\mathfrak{R}_{1}$.

\subsection{Theorem}

Let $\Re_{1}$ and $\mathfrak{R}_{2}$ be any two semi $\mathrm{HX}$ rings on the semi rings $\mathrm{R}_{1}$ and $\mathrm{R}_{2}$ respectively. Let $\mathrm{f}: \mathfrak{R}_{1} \rightarrow \mathfrak{R}_{2}$ be an anti homomorphism onto semi HX rings. Let $\mathrm{H}$ be an intuitionistic fuzzy subset of $R_{1}$. Let $\lambda^{\mathrm{H}}$ be an intuitionistic fuzzy semi HX ring of $\Re_{1}$ then $\mathrm{f}\left(\lambda^{\mathrm{H}}\right)$ is an intuitionistic fuzzy semi $\mathrm{HX}$ ring of $\mathfrak{R}_{2}$, if $\lambda^{\mathrm{H}}$ has a supremum property and $\lambda^{\mathrm{H}}$ is f-invariant.

\section{Proof}

Let $\mathrm{H}=\left\{\langle\mathrm{x}, \mu(\mathrm{x}), \eta(\mathrm{x})\rangle / \mathrm{x} \in \mathrm{R}_{1}\right\}$ be an intuitionistic fuzzy sets defined on a semi ring $\mathrm{R}_{1}$. Then, $\lambda^{\mathrm{H}}=\left\{\left\langle\mathrm{X}, \lambda^{\mu}(\mathrm{X}), \lambda^{\eta}(\mathrm{X})\right\rangle / \mathrm{X} \in \mathfrak{R}_{1}\right\}$ be an intuitionistic fuzzy semi HX ring of a semi HX ring $\Re_{1}$. Then, $\mathrm{f}\left(\lambda^{\mathrm{H}}\right)=\left\{\left\langle\mathrm{f}(\mathrm{X}), \mathrm{f}\left(\lambda^{\mu}\right)(\mathrm{f}(\mathrm{X})), \mathrm{f}\left(\lambda^{\eta}\right)(\mathrm{f}(\mathrm{X}))\right\rangle / \mathrm{X} \in \mathfrak{R}_{1}\right\}$. 


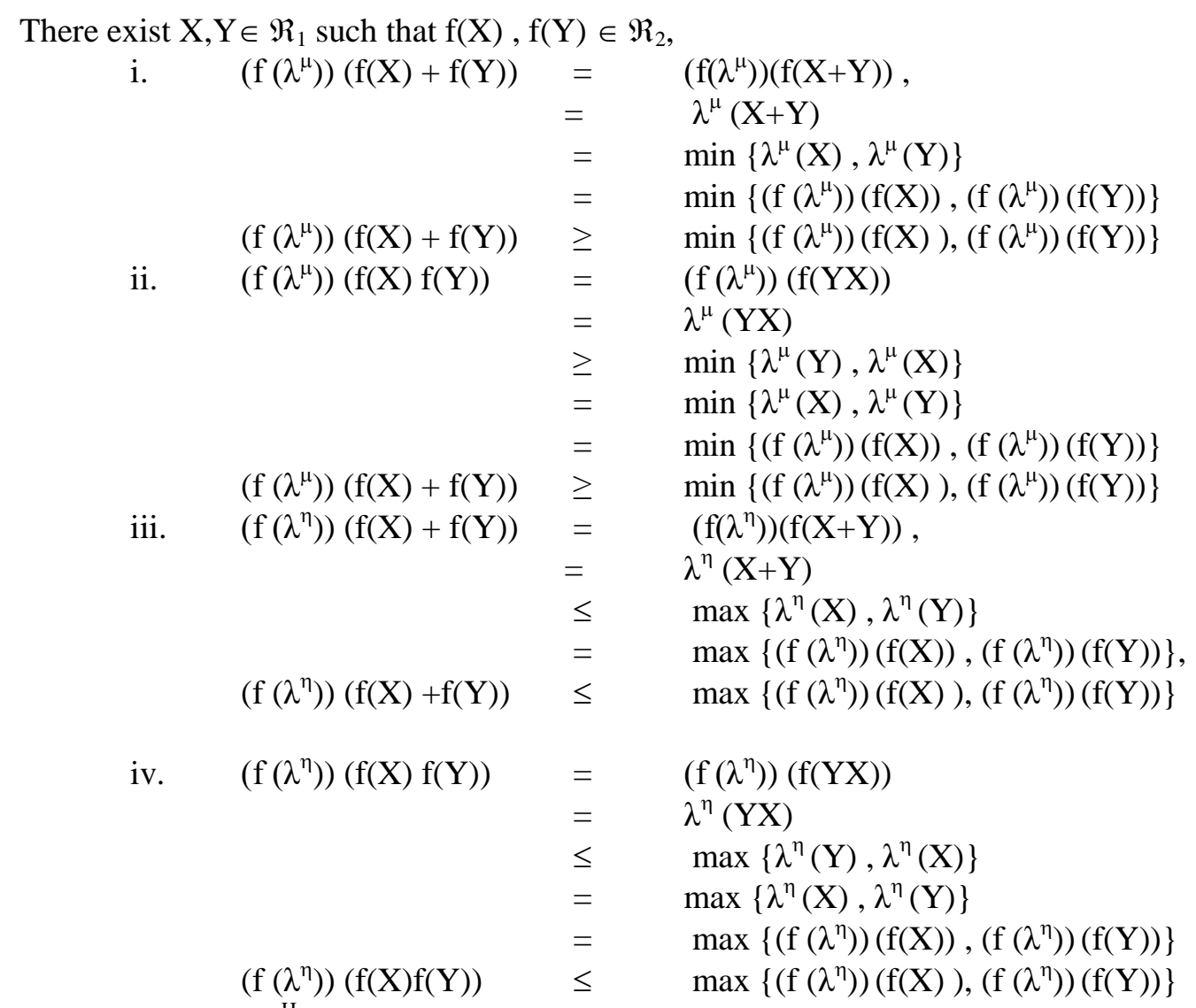

Hence, $\mathrm{f}\left(\lambda^{\mathrm{H}}\right)$ is an intuitionistic fuzzy semi HX ring of $\mathfrak{R}_{2}$.

\subsection{Theorem}

Let $\Re_{1}$ and $\Re_{2}$ be any two semi HX rings on $R_{1}$ and $R_{2}$ respectively. Let $f: \Re_{1} \rightarrow \Re_{2}$ be an anti homomorphism on semi HX rings. Let $\mathrm{G}$ be an intuitionistic fuzzy subset of $\mathrm{R}_{2}$. Let $\gamma^{\mathrm{G}}$ be an intuitionistic fuzzy semi HX ring of $\mathfrak{R}_{2}$, then $\mathrm{f}^{-1}\left(\gamma^{\mathrm{G}}\right)$ is an intuitionistic fuzzy semi HX ring of $\mathfrak{R}_{1}$.

\section{Proof}

Let $\mathrm{G}=\left\{\langle\mathrm{y}, \alpha(\mathrm{y}), \beta(\mathrm{y})\rangle / \mathrm{y} \in \mathrm{R}_{2}\right\}$ be an intuitionistic fuzzy sets defined on a semi ring $\mathrm{R}_{2}$. Then, $\gamma^{\mathrm{G}}=\left\{\left\langle\mathrm{Y}, \gamma^{\alpha}(\mathrm{Y}), \gamma^{\beta}(\mathrm{Y})\right\rangle / \mathrm{Y} \in \mathfrak{R}_{2}\right\}$ be an intuitionistic fuzzy semi HX ring of a semi HX ring $\Re_{2}$. Then, $\mathrm{f}^{-1}\left(\gamma^{\mathrm{G}}\right)=\left\{\left\langle\mathrm{X}, \mathrm{f}^{-1}\left(\gamma^{\alpha}\right)(\mathrm{X}), \mathrm{f}^{-1}\left(\gamma^{\beta}\right)(\mathrm{X})\right\rangle / \mathrm{X} \in \mathfrak{R}_{1}\right\}$.

For any $X, Y \in \mathfrak{R}_{1}, f(X), f(Y) \in \mathfrak{R}_{2}$,

\begin{tabular}{|c|c|c|c|}
\hline \multirow[t]{5}{*}{ i. } & $\left(\mathrm{f}^{-1}\left(\gamma^{\alpha}\right)\right)(\mathrm{X}+\mathrm{Y})$ & $=$ & $\gamma^{\alpha}(f(X+Y))$ \\
\hline & & $=$ & $\gamma^{\alpha}(\mathrm{f}(\mathrm{X})+\mathrm{f}(\mathrm{Y}))$ \\
\hline & & $\geq$ & $\min \left\{\gamma^{\alpha}(\mathrm{f}(\mathrm{X})), \gamma^{\alpha}(\mathrm{f}(\mathrm{Y}))\right\}$ \\
\hline & & $=$ & $\min \left\{\left(\mathrm{f}^{-1}\left(\gamma^{\alpha}\right)\right)(\mathrm{X}),\left(\mathrm{f}^{-1}\left(\gamma^{\alpha}\right)\right)(\mathrm{Y})\right\}$ \\
\hline & $\left(\mathrm{f}^{-1}\left(\gamma^{\alpha}\right)\right)(\mathrm{X}+\mathrm{Y})$ & $\geq$ & $\min \left\{\left(\mathrm{f}^{-1}\left(\gamma^{\alpha}\right)\right)(\mathrm{X}),\left(\mathrm{f}^{-1}\left(\gamma^{\alpha}\right)\right)(\mathrm{Y})\right\}$ \\
\hline \multirow[t]{6}{*}{ ii. } & $\left(f^{-1}\left(\gamma^{\alpha}\right)\right)(X Y)$ & $=$ & $\gamma^{\alpha}(\mathrm{f}(\mathrm{XY}))$ \\
\hline & & $=$ & $\gamma^{\alpha}(f(Y) f(X))$ \\
\hline & & $\geq$ & $\min \left\{\gamma^{\alpha}(f(Y)), \gamma^{\alpha}(f(X))\right\}$ \\
\hline & & $=$ & $\min \left\{\gamma^{\alpha}(f(X)), \gamma^{\alpha}(f(Y))\right\}$ \\
\hline & & $=$ & $\min \left\{\left(\mathrm{f}^{-1}\left(\gamma^{\alpha}\right)\right)(\mathrm{X}),\left(\mathrm{f}^{-1}\left(\gamma^{\alpha}\right)\right)(\mathrm{Y})\right\}$ \\
\hline & $\left(f^{-1}\left(\gamma^{\alpha}\right)\right)(X Y)$ & $\geq$ & $\min \left\{\left(\mathrm{f}^{-1}\left(\gamma^{\alpha}\right)\right)(\mathrm{X}),\left(\mathrm{f}^{-1}\left(\gamma^{\alpha}\right)\right)(\mathrm{Y})\right\}$ \\
\hline \multirow[t]{5}{*}{ iii. } & $\left(\mathrm{f}^{-1}\left(\gamma^{\beta}\right)\right)(\mathrm{X}+\mathrm{Y})$ & $=$ & $\gamma^{\beta}(\mathrm{f}(\mathrm{X}+\mathrm{Y}))$ \\
\hline & & $=$ & $\gamma^{\beta}(\mathrm{f}(\mathrm{X})+\mathrm{f}(\mathrm{Y}))$ \\
\hline & & $\leq$ & $\max \left\{\gamma^{\beta}(\mathrm{f}(\mathrm{X})), \gamma^{\beta}(\mathrm{f}(\mathrm{Y}))\right\}$ \\
\hline & & $=$ & $\max \left\{\left(\mathrm{f}^{-1}\left(\gamma^{\beta}\right)\right)(\mathrm{X}),\left(\mathrm{f}^{-1}\left(\gamma^{\beta}\right)\right)(\mathrm{Y})\right\}$ \\
\hline & $\left(\mathrm{f}^{-1}\left(\gamma^{\beta}\right)\right)(\mathrm{X}+\mathrm{Y})$ & $\leq$ & $\max _{\beta}\left\{\left(\mathrm{f}^{-1}\left(\eta^{\beta}\right)\right)(\mathrm{X}),\left(\mathrm{f}^{-1}\left(\gamma^{\beta}\right)\right)(\mathrm{Y})\right\}$. \\
\hline & & & $\gamma^{\beta}(\mathrm{f}(\mathrm{XY}))$ \\
\hline
\end{tabular}




$\begin{array}{rll} & = & \gamma^{\beta}(\mathrm{f}(\mathrm{Y}) \mathrm{f}(\mathrm{X})) \\ & \leq & \max \left\{\gamma^{\beta}(\mathrm{f}(\mathrm{Y})), \gamma^{\beta}(\mathrm{f}(\mathrm{X}))\right\} \\ & = & \max \left\{\gamma^{\beta}(\mathrm{f}(\mathrm{X})), \gamma^{\beta}(\mathrm{f}(\mathrm{Y}))\right\} \\ & = & \max \left\{\left(\mathrm{f}^{-1}\left(\gamma^{\beta}\right)\right)(\mathrm{X}),\left(\mathrm{f}^{-1}\left(\gamma^{\beta}\right)\right)(\mathrm{Y})\right\} \\ \left(\mathrm{f}^{-1}\left(\gamma^{\beta}\right)\right)(\mathrm{XY}) & \leq & \max \left\{\left(\mathrm{f}^{-1}\left(\eta^{\beta}\right)\right)(\mathrm{X}) \mathrm{I},\left(\mathrm{f}^{-1}\left(\gamma^{\beta}\right)\right)(\mathrm{Y})\right\}\end{array}$

Hence, $f^{-1}\left(\gamma^{G}\right)$ is an intuitionistic fuzzy semi HX ring of $\mathfrak{R}_{1}$.

\section{CONCLUSION}

This paper used to enrich our focus towards intuitionistic fuzzy semi HX ring and its properties. It help us to study the applications of intuitionistic Fuzzy semi HX ring later.

\section{ACKNOWLEDGEMENTS}

We, the authors would like to express our sincere gratitude to all our fellow friends for their extended help to make this research article as a successful one. Also we thank the reviewers of this journal for their kind consideration of this article to publish.

\section{REFERENCES}

[1]. Atanassov.K.T, intuitionistic fuzzy sets,Fuzzy Sets and System 20 (1986) ,pp 87-96.

[2]. Bing-xueYao and Yubin-Zhong, Upgrade of algebraic structure of ring, Fuzzy information and Engineering (2009)2:219-228.

[3]. Bing-xueYao and Yubin-Zhong, The construction of power ring, Fuzzy information and Engineering (ICFIE), ASC 40,pp.181-187,2007.

[4]. Dheena.P and Mohanraaj.G, On intuitionistic fuzzy $\mathrm{k}$ ideals of semi rings, International Journal of computational cognition, Volume 9, No.2, 45-50, June 2011.

[5]. Li Hong Xing, HX group, BUSEFAL, 33(4), 31-37, October 1987.

[6]. Li Hong Xing, HX ring, BUSEFAL, 34(1) 3-8, January 1988.

[7]. Liu.W.J., Fuzzy invariant subgroups and fuzzy ideals, Fuzzy sets and systems, 8, 133 - 139.

[8]. Muthuraj.R, Manikandan K.H., Sithar Selvam.P.M., Intuitionistic Q-fuzzy normal HX group, International Journal of Mathematical Archive, Vol.2, no10., pp 1828-1832.

[9]. Muthuraj.R, Manikandan K.H., Intuitionistic Q-fuzzy HX subgroup and $<\alpha, \beta>$ level sub HX groups, CIIT International DOI - FS 122011010.

[10]. Rosenfeld. A., Fuzzy groups, J.Math.Anal., 35(1971),512-517.

[11].Zadeh.L.A., Fuzzy sets, Information and control,8,338-353.

[12]. Zhong YB (1995) The structure of HX-ring on a class of ring. Fuzzy Systems and Mathematics 9(4) pp.7377. 


\section{AUTHOR'S BIOGRAPHY}

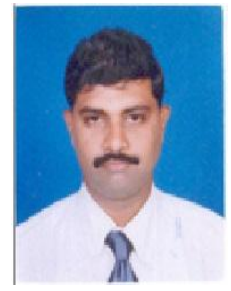

Dr. R. Muthuraj, Assistant professor of Mathematics has been working in H.H.Rajahs college, Pudukottai since 2010. He completed his M.Sc., and M.Phil., in Madura College with distinction. He completed his Ph.D., in Alagappa University, Karaikudi. He has published more than 75 papers in the fields of fuzzy algebra and fuzzy topology. He has produced $10 \mathrm{Ph}$. D., candidates in the field of fuzzy algebra in Bharathidasan university and in Anna university. Now 6 candidates pursuing Ph.D., under his guidance. He is an active reviewer for many journals. He has written 2 books for Engineering Mathematics.

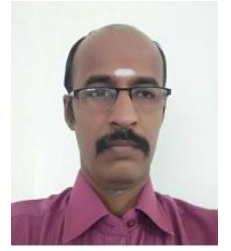

Mr. M.S. Muthuraman, Associate professor of Mathematics has been working in PSNACET, Dindigul since 2004. He has completed his M.Sc., at Sourashtra College and M.Phil., in Madurai Kamaraj University, Madurai. He is pursuing his Ph.D., in Bharathidasan University, under the guidance of Dr. R. Muthuraj. He has published more than 25 papers in the fields of fuzzy algebra. He has written 4 books for Engineering Mathematics.

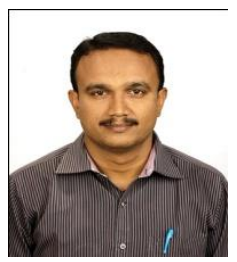

K.H.Manikandan, is working as an Assistant Professor in Department of Mathematics, P.S.N.A. College of Engg., \& Tech., Dindigul, Tamilnadu, India, with 16 years of teaching experience. He has published 15 National and International journals. He has completed his doctoral degree under the guidance of Dr. R. Muthuraj at Anna University in October 2015. He is an active reviewer in Annals of Fuzzy Mathematics and Informatics and Global Research and Development journals. He is very much interested in applications of fuzzy algebra.

Citation: R. Muthuraj et al., "Intuitionistic Fuzzy Semi HX Ring", International Journal of Scientific and Innovative Mathematical Research, vol. 6, no. 5, p. 1-13, 2018., http://dx.doi.org/10.20431/23473142.0605001

Copyright: () 2018 Authors. This is an open-access article distributed under the terms of the Creative Commons Attribution License, which permits unrestricted use, distribution, and reproduction in any medium, provided the original author and source are credited. 